Published in final edited form as:

Chem Mater. 2017 November 14; 29(21): 9120-9132. doi:10.1021/acs.chemmater.7b02845.

\title{
Three-Dimensional Microphase Separation and Synergistic Permeability in Stacked Lipid-Polymer Hybrid Membranes
}

\author{
Minjee Kang ${ }^{\dagger}$, Byeongdu Lee $^{\ddagger}$, and Cecilia Leal ${ }^{\star}, \dagger$ \\ tDepartment of Materials Science and Engineering, University of Illinois at Urbana-Champaign, \\ Urbana, Illinois 61801, United States \\ ¥X-ray Science Division, Argonne National Laboratory, Argonne, Illinois 60439, United States
}

\begin{abstract}
We present new structures of soft-material thin films that augment the functionality of substratemediated delivery systems. A hybrid material composed of phospholipids and block copolymers adopts a multilayered membrane structure supported on a solid surface. The hybrid films comprise intentional intramembrane heterogeneities that register across multilayers. These stacked domains convey unprecedented enhancement and control of permeability of solutes across micrometerthick films. Using grazing incidence X-ray scattering, phase contrast atomic force microscopy, and confocal microscopy, we observed that in each lamella, lipid and polymers partition unevenly within the membrane plane segregating into lipid- or polymer-rich domains. Interestingly, we found evidence that like-domains align in registry across multilayers, thereby making phase separation three-dimensional. Phase boundaries exist over extended length scales to compensate the height mismatch between lipid and polymer molecules. We show that microphase separation in hybrid films can be exploited to augment the capability of drug-eluting substrates. Lipid-polymer hybrid films loaded with paclitaxel show synergistic permeability of drug compared to singlecomponent counterparts. We present a thorough structural study of stacked lipid-polymer hybrid membranes and propose that the presence of registered domains and domain boundaries impart enhanced drug release functionality. This work offers new perspectives in designing thin films for controlled delivery applications.
\end{abstract}

\section{Graphical Abstract}

\footnotetext{
*Corresponding Author: cecilial@illinois.edu.

Supporting Information

The Supporting Information is available free of charge on the ACS Publications website at DOI: 10.1021/acs.chemmater.7b02845. CLSM images of hybrid films doped with NBD-DPPE; GISAXS data of hybrid films; AFM images of hybrid and pure films without Paclitaxel drugs; AFM images of hybrid and pure films with Paclitaxel drugs; GIWAXS data of Paclitaxel incorporating films; CLSM images of hybrid films with Paclitaxel-Oregon Green 488; Paclitaxel release profiles from hybrid films with different molar percentages of Paclitaxel incorporated; WAXS data of lipid, polymer, and hybrid films with different molar percentages of Paclitaxel incorporated; optical microscopy images of hybrid and pure films with Paclitaxel drugs; Paclitaxel loading efficiencies for lipid, polymer, and hybrid films with different molar percentages of Paclitaxel incorporated (PDF)

The authors declare no competing financial interest.
} 


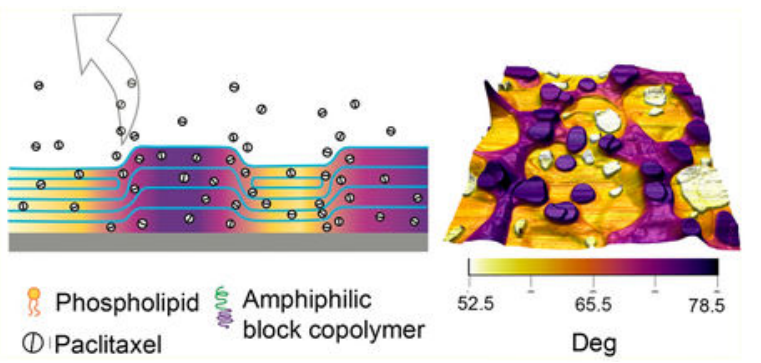

\section{INTRODUCTION}

There was a time when the cell membrane was perceived as an assembly of randomly distributed phospholipids and membrane proteins. ${ }^{1}$ However, extensive research on cell membranes has led to a change in such perception, and it is now widely acknowledged that cell membranes comprise local heterogeneities with distinct compositions and structures. ${ }^{2-6}$ The lateral heterogeneities in cell membranes with tunable dynamic properties have been shown to engage in selective partitioning of membrane proteins such as $\mathrm{K}^{+},{ }^{7} \mathrm{Na}^{+},{ }^{8} \mathrm{Ca}^{+}$ion ${ }^{9}$ channels and linker for activation of T cells (LAT), ${ }^{10}$ regulating diffusion of proteins, ${ }^{10}$ and entry of viruses. ${ }^{11-13}$

Inspired by the functional roles of those local heterogeneities in cell membranes, selfassembly structures that mimic in-plane segregated domains have been explored for a number of applications. ${ }^{14-17}$ A noteworthy approach is the mixing of phospholipids and amphiphilic block copolymers into a self-assembled complex. Beyond self-assembly of single type of amphiphiles that has been established, ${ }^{18-20}$ concurrent self-assembly of multiple amphiphiles has emerged as an approach to build more complex architectures with functionalities not achievable by single components. ${ }^{21-24}$ Upon certain conditions, lipid and polymer molecules self-organize into a mixed hybrid membrane in the form of vesicles, ${ }^{25-31}$ monolayers at the air-water interface, ${ }^{16}$ or solid-supported bilayers ${ }^{32}$ where phase-separated domains are often found. In this study, we demonstrate that hybrid membranes self-assemble into phase-separated stacked domains on a solid support.

Interestingly, phase-separated domains in these biomimetic hybrid membranes resemble the lateral heterogeneities in cell membranes in terms of their functions: controlling the localization and diffusion rates of molecules embedded into the membranes, ${ }^{15,16,33}$ and controlling the entry of materials into cells. ${ }^{34}$ For example, Olubummo et al. demonstrated that polymer-functionalization of $\mathrm{CdSe}$ nanoparticles could guide the particles to selectively locate within polymer domains over lipid domains in a mixed lipid-polymer monolayer. ${ }^{15} \mathrm{In}$ a similar vein, controlling fluidity of the lipid-polymer membrane was shown to direct the preferential insertion of membrane proteins into one domain over the other. ${ }^{16}$

Previous studies on lipid-polymer hybrid membranes have demonstrated the possibilities of the hybrids to serve as a new functional membrane platform. It is especially promising that the domains can be engineered to have the desired size, morphologies, and mechanical properties. ${ }^{35,36}$ Studies on hybrid membranes so far have been focused in two-dimensional single membranes. ${ }^{15,16,32,33,37}$ However, in nature there exists a wide variety of membrane 
systems where the three-dimensional (3D) structure of lipid membranes mediates functionality and adaptability to external conditions. ${ }^{38,39}$ One interesting example is the stacked lipid membranes (thylakoids) in the chloroplast of plant cells. The lateral heterogeneity of thylakoid membranes ${ }^{40}$ denotes the nonrandom distribution of photosystem II, light-harvesting complex II, photosystem I, and ATP synthase complexes in the thylakoids. It is speculated that these lateral heterogeneities stack up (and unstack) in registry as a response to changing environmental conditions (e.g., moisture and light) ${ }^{41,42}$ as a nature's effort to optimize the light capture and energy transport efficiencies.

Such indications of the membrane stacked domains in nature, being present in 3D membrane assemblies and exerting an influence on the functions, intrigued us to work on stacked membranes of lipid-polymer hybrids with phase-separated domains. While lipid-polymer hybrid single membranes with lateral phase-separated domains have been studied before, domain stacking events in 3D and their exploitation for membrane permeability have not been investigated before.

Our goal in this study was two-fold. First, we constructed multilayered lipid-polymer hybrid membranes and characterized the structure focusing on intralayer and interlayer domain structures. Second, we tested the functionality of hybrid films as a new class of substratemediated drug delivery platform. Substrate- (or surface-) mediated drug delivery is a novel research direction in the field of biomedical engineering and health care devices. ${ }^{43} \mathrm{In}$ contrast to systemic particulate drug delivery, surface-mediated drug delivery delivers drugs at the localized treatment site, allowing great spatial control over the release of therapeutic cargo. ${ }^{44}$ Developing functional matrices ${ }^{43-45}$ is crucial for a broad range of biomedical applications including drug eluting stents, ${ }^{46,47}$ therapeutic implants, ${ }^{20,48}$ and tissue engineering. ${ }^{49-51}$

The second part of the work was followed by interesting observations from the first part that presented the possibility of hybrid films serving as active drug release matrices beyond simple depots of the embedded drug molecules. In each plane of bilayers, mixtures of lipids and polymers phase-separate into different domains induced by a hydrophobic mismatch between lipid and polymer chains. The steep hydrophobic mismatch between chains results in the propagation of domain phase boundaries over large length scales. Interestingly, the phase-separated domains between neighboring layers were shown to align on top of each other, representing a smectic ordering that continues across the stacks of layers. Analogous behavior has been observed in stacked bilayers of ternary lipid mixtures studied by Tayebi et al. ${ }^{52}$ These observations prompted our conjecture that domains aligned in registry, as well as large domain boundary areas in the hybrid films, may affect the transport of drugs across films. Indeed, we found an enhanced permeability effect of hydrophobic drugs incorporated into hybrid films that we attribute to membrane domain structures.

\section{MATERIALS AND METHODS}

\section{Materials.}

1,2-Dipalmitoyl-sn-glycero-3-phosphocholine (16:0 PC or DPPC) and 1,2-dipalmitoyl-snglycero-3-phosphoethanolamine-N-(7-nitro-2-1,3-benzoxadiazol-4-yl) (16:0 NBD PE or 
NBD-DPPE) were purchased from Avanti Polar Lipids (Alabaster; AL, USA). Amphiphilic diblock copolymer, poly(butadiene- $b$-ethylene oxide) (PBD- $b$-PEO), was purchased from Polymer Source, Inc. (Quebec, Canada). The catalog number is P19015-BdEO. Its average molecular weight (Mn) was reported to be 4000 with PBD block (rich in 1,4 microstructure) 2500 and PEO block 1500, respectively. The reported polydispersity was 1.06. In this work, we selected PBD- $b$-PEO since the previous studies successfully demonstrated that the mixtures of phospholipids/PBD- $b$-PEO form phase-separated hybrid vesicles with domains. 25,35 For biomedical applications, the use of biocompatible polymer blocks such as polyisobutylene or polyoxazoline is advised. Paclitaxel, its fluorescent conjugate PaclitaxelOregon Green 488, and DilC18(5) oil (1,1' -dioctadecyl-3,3,3',3' -

tetramethylindodicarbocyanine perchlorate) were purchased from Thermo Fisher Scientific (Waltham; MA, USA). All solvents used were of high performance liquid chromatography (HPLC) grade and purchased from Sigma-Aldrich (St Louis; MO, USA). All chemicals and materials were used as received.

\section{Preparation of Multilamellar Films.}

Multilamellar films composed of phospholipids, block copolymers, and their hybrid were prepared by either drop-casting or spin-coating methods. Typically, drop-cast films have thicknesses ranging from 5 to $20 \mu \mathrm{m}$ and spin-coated films range from 200 to $500 \mathrm{~nm}$. Samples used for substrate-mediated drug delivery were prepared by drop-casting so that there is control over the exact amount and ratio of materials being incorporated. Samples for CLSM and GISWAXS were also prepared by drop-casting. In the case of samples prepared for AFM measurements, spin-coating was used to minimize AFM cantilever crashing onto rough sample surfaces. Lipids and polymers were dissolved in chloroform. Paclitaxel and its fluorescent conjugate were dissolved in ethanol. A stock solution was prepared with the desired ratio of each component in mixtures including lipids, polymers, or paclitaxel molecules (concentration, 10-25 mg/mL; solvent, chloroform/ethanol 4:1 volume ratio). The stock solution was either dropped onto the substrate followed by a solvent evaporation under a fume hood or spin-coated at $1000 \mathrm{rpm}$ for $30 \mathrm{~s}$. For complete solvent evaporation, samples were put into the vacuum desiccator overnight. Upon solvent evaporation, the amphiphilic nature of lipid and polymer molecules leads to self-assembly into well-organized multilamellar films. The substrates used in the experiments were coverslips with a diameter of $15 \mathrm{~mm}$ purchased from MatTek Corporation (Ashland; MA, USA). Before use, the coverslips were washed with ethanol and Milli-Q water for 15 min respectively in an ultrasonic bath and dried with a nitrogen gun. For AFM and X-ray scattering experiments, to ensure that substrates do not affect the overall structure of films and interpretation of the data, samples were also prepared onto cleaved Si wafers $\left.\left(\sim 1 \mathrm{~cm}^{2}\right) ;<100\right\rangle$ type that was purchased from University Wafer Inc. (South Boston; MA, USA). We did not observe noticeable structural differences between samples prepared onto coverslips and silica substrates.

\section{Structure Characterization of Multilamellar Films.}

Confocal Laser Scanning Microscopy (CLSM)._CLSM images were obtained in water at room temperature to (i) check the presence of phase separated domains and (ii) identify the drug distribution in films using a Zeiss LSM 700 microscope (Carl Zeiss AG, 
Germany) with $20 \times / 0.8$ DIC, $40 \times / 1.3$ DIC, and $63 \times / 1.4$ Oil DIC objectives. A concentration of $0.1 \mathrm{~mol} \%$ of 16:0 NBD-DPPE (Ex/Em $460 \mathrm{~nm} / 535 \mathrm{~nm}$ ) was used to label the lipid-rich phase and $0.2 \mathrm{~mol} \%$ of Paclitaxel-Oregon Green $488(\mathrm{Ex} / \mathrm{Em} 488 \mathrm{~nm} / 518 \mathrm{~nm})$ was used as a fluorescent model drug to represent the drug distribution in films. A concentration of 0 . One mol \% of DilC18(5) oil was used in combination with Paclitaxel-Oregon Green 488. Images were processed using the Zen software (Carl Zeiss AG). For samples stained with single dye molecules, the pinhole was set to 1 Airy unit. For multichannel experiments, the Paclitaxel-Oregon Green channel was set to 1 Airy unit and the other channel was adjusted to match the optical slice thickness as the Paclitaxel-Oregon Green channel.

Atomic Force Microscopy (AFM).-Simultaneous height (topography) and phase imaging were performed on lipid, polymer, and hybrid films to (i) obtain nanometer-scale information about the surface structure and to (ii) identify different component phases of hybrid films. Especially, the phase boundaries where lipid domains and polymer domains meet were carefully investigated. For all measurements, a MFP-3D AFM (Asylum Research; CA, USA) was operated in tapping mode in air at room temperature. Tap300AL-G cantilevers were used with a resonance frequency of $300 \mathrm{kHz}$ and a force constant of $40 \mathrm{~N} / \mathrm{m}$ (Budget Sensors; Sofia, Bulgaria).

\section{Grazing-Incidence Small/Wide-Angle X-ray Scattering (GISWAXS).-GISAXS}

and GIWAXS experiments were conducted to study the multilamellar film structure and lipid hydrocarbon tail ordering as well as drug crystallization, respectively. The experiments were carried out at in-house X-ray setup (custom built with help of Forvis Technologies, CA, USA) and at 12-ID-B beamline, Advanced Photon Source (APS), Argonne National Laboratory. A custom built (with Forvis Technologies, Santa Barbara) equipment composed of a Xenocs GeniX3D Cu Ka ultralow divergence X-ray source $(8 \mathrm{keV})$ was used, with a divergence of $1.3 \mathrm{mrad}$. The humidity and temperature control chambers were built by Forvis Technologies. At the 12-ID-B beamline APS, a $14 \mathrm{keV} \mathrm{X-ray} \mathrm{beam} \mathrm{was} \mathrm{focused} \mathrm{on} \mathrm{a}$ $50 \times 10 \mu \mathrm{m}^{2}(\mathrm{H} \times \mathrm{V})$ area at an incident angle $0.05-0.2^{\circ}$. Pilatus2M (Dectris) and PerkinElmer XRPad 4343F detectors were used for GISAXS and GIWAXS measurements, respectively. The sample-to-detector distance was calibrated using a silver behenate powder standard. The specular beam intensity was attenuated along the $\mathrm{z}$ axis with a strip beam stop. The sample to detector distance for GISAXS was either 2 or $3.6 \mathrm{~m}$, and that for GIWAXS was about $0.15 \mathrm{~m}$. Multiple measurements were carried on the same sample in a humidity chamber (relative humidity >95\%), varying the incidence angle to determine the most appropriate operating angle. Experiments at the APS were performed at room temperature, and in-house experiments were performed at different temperatures $\left(25^{\circ} \mathrm{C}, 37^{\circ} \mathrm{C}\right.$, and $\left.45^{\circ} \mathrm{C}\right)$. The measured scattering patterns were analyzed using NIKA and IRENA, Igor-based package of tools for scattering data analysis. ${ }^{53}$

\section{Paclitaxel Release Study.}

Film samples prepared onto $15 \mathrm{~mm}$ glass coverslips $(n=3)$ were placed into a 24 -well cell culture plate with a well diameter of $15.6 \mathrm{~mm}$. During entire release experiments, the well plate was shaken using a plate shaker with a shaking speed of $100 \mathrm{rpm}$ at room temperature. In each well, $1 \mathrm{~mL}$ of release medium PBS/ethanol 90/10 (\% v/v) was added which has been 
used in previous work ${ }^{54}$ to achieve practical sink conditions for paclitaxel, a poorly watersoluble drug (solubility: $0.3 \mu \mathrm{g} / \mathrm{mL}^{55}$ ). At predetermined time points, the release medium was completely withdrawn and replaced with the same volume of fresh medium, maintaining the sink conditions throughout the release experiments. For the first $5 \mathrm{~min}$, surface-attached paclitaxel not incorporated inside the membrane was removed by exposing and washing in the release medium. The drug loading efficiency was determined by the following equation: loading efficiency $(\%)=$ (total amount of drug - free drug in the medium)/total amount of drug $\times 100$. The amount of paclitaxel released was quantified using a high-performance liquid chromatography (HPLC). HPLC analysis was conducted by Shimadzu LC system (LC-20AT) connected with a PDA detector (SPD-M20A). A Phenomenex Kinetex Ph-hexyl column (5 $\mu \mathrm{m}, 100 \mathrm{~mm} \times 4.6 \mathrm{~mm}$ ) was used for analysis. Acetonitrile/water 60/40 (\% v/v) served as a mobile phase, eluting the paclitaxel peak approximately at $1.3 \mathrm{~min}$ with a flow rate $1.5 \mathrm{~mL} / \mathrm{min}$ from an injection volume of $20 \mu \mathrm{L}$ with UV/vis detector recorded at $229 \mathrm{~nm}$. The area of the paclitaxel peak was integrated and compared to a standard calibration curve obtained in the concentration range of 0.1-10 $\mu \mathrm{g} / \mathrm{mL}$ (regression value $R^{2}=0.9994871$ ). The detection limit of paclitaxel in the release medium was $0.08 \mu \mathrm{g} / \mathrm{mL}$.

\section{RESULTS AND DISCUSSION}

\section{Phase-Separation in Multilamellar Hybrid Films.}

To investigate the mixing behavior of polymers and lipids, we employed CLSM to films deposited on a solid support. NBDDPPE fluorescent probes were added to the lipid/polymer mixture as a means to distinguish between lipid (DPPC) and polymer (PBD- $b$-PEO) phases as they are known to favorably partition into DPPC domains. Such preference was reported previously for systems of model membranes composed of saturated lipids (e.g., DPPC) and unsaturated lipids with cholesterol, ${ }^{56}$ and lipid-polymer hybrid giant unilamellar vesicles (DPPC/PBD- $b$-PEO). ${ }^{27}$ Figure 1A shows the series of the hybrid film images taken at different focal depth $(Z)$ positions. Coexistence of lipid and polymer phases was readily observed from binary fluorescence patterns. In each image, the green regions indicate phase separated domains enriched in DPPC lipids, while the dark regions show the domains enriched in PBD- $b$-PEO polymers. The size of the lipid domains was on the order of several microns when the entire film thickness was on the order of $20 \mu \mathrm{m}$ (measured by CLSM). Such observation was carried out at room temperature. The domain sizes would vary upon temperature changes. ${ }^{57,58}$ The lipid domains display either quasi-circular or elongated line shapes. These types of morphology have been previously observed in solid-phase lipid domains. ${ }^{59}$

Additional fluorescence patterns obtained from hybrid films can be found in Figure S1 (Supporting Information). Surprisingly, the fluorescence patterns were almost identical at different depths of the films, suggesting that there is alignment of like-domains on top of each other across the layers. It should be noted that the stacking of domains applies only to the optically observable domains ( $\mu \mathrm{m}$ scale). It is possible that optically unresolvable small domains randomly locate across multilayers. 
Figure 1B schematically depicts the possibilities of domain alignment across stacked bilayers. When lipid (or polymer) domains stack in registry onto lipid (or polymer) domains, a schematic configuration of the domain alignment would be close to Figure 1B-1. In contrast, if the domains randomly stack not coupling across layers, the cross-section of the films would correspond to Figure 1B-2. The depth-resolved CLSM images suggest the domain alignment to be the case of Figure 1B-1.

To better understand the domain alignment in the direction normal $(z)$ to the membrane, we carried out GISAXS experiments on the stacked membranes. Figure. 1C shows the twodimensional GISAXS data (left) and the corresponding radial averaged intensity versus $\mathrm{q}$ profiles (right) of the stacked membranes. The 2D GISAXS data displays an array of distinct and periodic patterns indicative of a multilamellar arrangement within the film depth direction $\left(q_{z}\right)$. The fact that the diffraction patterns have higher intensity along $q_{z}$ further suggests that multilayers are highly oriented parallel to the substrate. The 1D line cut profiles show the diffraction pattern of a lamellar phase which can be recognized by a series of peaks at equal interpeak distance. The interlayer spacing of the lamellar structure can be calculated from the position of the peaks by $a=2 \pi \mathrm{n} / q_{n}$, where $q_{n}$ denotes the position of the $n$th order peak. Sharp diffraction patterns from the pure lipid phase (red line) and the pure polymer phase (blue line) yield a lamellar spacing $a_{\text {lipid }}=55.6 \AA\left(q_{001}\right.$, lipid $\left.=0.113 \AA^{-1}\right)$ and $a_{\text {polymer }}=124 \AA\left(q_{001}\right.$, polymer $\left.=0.0506 \AA^{-1}\right)$, respectively. The lipid-polymer hybrid films showed two sets of diffraction peaks arising from the lipid lamellar phase and the polymer lamellar phase. The lamellar spacing of lipid phase in hybrid films increased to ${ }_{a} H_{\text {lipid }}=57.4 \AA$ compared to that of single-component lipid films $\left(a_{\text {lipid }}=55.6 \AA\right)$, while polymer phase in hybrid films maintained the same lamellar spacing $\mathrm{a}_{\text {polymer }}=a_{\text {polymer }}=$ $124 \AA$. An increase in the lamellar spacing $a^{H}{ }_{\text {lipid }}$ can be attributed from several factors including changes in the lipid chain configuration and membrane-membrane interactions of lipids. For example, long-range steric repulsion due to membrane fluctuations (undulations resulting from increased flexibility of bilayers) would lead to an increased lamellar spacing of lipids. ${ }^{60-62}$ We observed structural changes of lipids at the molecular level using GIWAXS (details presented at later sections), which substantiates increased flexibility of bilayers. Upon the presence of polymer, the long-range ordering of lipid headgroups was lost, and some portion of lipid tails were shown to rearrange their configuration from tilted chains to untilted chains along the bilayer normal.

The two sets of lamellar repeat distances in the hybrid films would most likely not be as well-resolved if the domains would stack randomly or alternating (lipid after polymer or polymer after lipid) between neighboring layers. Figure S2 (Supporting Information) shows GISAXS data obtained for the hybrid films equilibrated at different temperatures $\left(25^{\circ} \mathrm{C}\right.$, $37^{\circ} \mathrm{C}$, and $50^{\circ} \mathrm{C}$ ). We observed a reversible response of the lipid and polymer phases upon heating and cooling cycles further supporting the presence of in-plane domains that are registered across layers. The GISAXS data combined with the CLSM images are only consistent with a picture where lateral in-plane lipid domains (or polymer domains) align with the lipid domains (or polymer domains) across the water layer in the neighboring bilayers (illustration Figure 1B-1). We speculate that interlayer stacking of alike domains may originate from the different hydrogen bonding networks between the hydrophilic PEO 
chains of PBD- $b$-PEO and the hydrophilic headgroups of DPPC lipids, and a concomitant hydrophobic effect drive for registry.

It is noteworthy that the repeat distances obtained for polymer (124 ̊) and lipid (57.4 $\AA$ ) bilayers are dramatically different. We find it intriguing that despite the large height mismatch between lipid and polymer molecules there is still a preference for the system to adopt a micro phase-separated state. The height mismatch at the phase boundary would cause a large hydrophobic surface being exposed to water, which is energetically unfavorable. The surface tension of hydrocarbon-water surfaces is reported ${ }^{63}$ to be 50 $\mathrm{erg} / \mathrm{cm}^{2}$. A thickness mismatch of only $1 \mathrm{~nm}$ results in an energetic cost per unit length of interface of $\sim 10 \mathrm{k} T / \mathrm{nm}$. To alleviate the energy of unfavorable hydrophobic interactions at the interface, elastic deformations of lipid and polymer chains should occur near the phase boundary. However, it is hard to imagine how such significant height mismatch would be compensated by membrane deformations in lipid-polymer hybrid films especially because the lipid components are expected to be in a gel state. ${ }^{64,65}$ The CLSM images could only provide qualitative hints on phase-separation of the hybrid films because of the resolution limit. To gain further information on the morphology of domains in hybrid films, we investigated the surface structures by means of topographical and phase contrast imaging in AFM.

\section{Phase Boundary Regions Induced by Hydrophobic Mismatch in Hybrid Films.}

The nanoscopic structures of the hybrid film could be clarified via simultaneous measurements of AFM height (topography) and phase data. AFM phase imaging allows the distinction between different material domains as the phase signals are sensitive to variations in material properties such as viscoelasticity and adhesion. ${ }^{66,67}$ Figure $2 \mathrm{~A}$ shows the AFM data obtained from hybrid films where the phase color contrast was overlaid onto the pseuso-3D construction of the height image. The AFM images on hybrid films presented interesting structures that could not be distinguished clearly from CLSM images. From the CLSM studies, we could identify a microphase separation behavior of polymers and lipids. The AFM phase images (Figure 2B,C) also indicate the presence of two distinctive islands of domains, which can be inferred from the color (phase) contrast. Interestingly, each prominent domain seems to be connected by intermediate regions. The light yellow colored domains (phase angle $70-80^{\circ}$ ) and the dark purple colored domains (phase angle 50-60 are thought to designate lipid and polymer domains, respectively. This was inferred from the morphology and height of each domain. The purple domains occupy a higher surface area on average, which agrees with the larger lamellar repeat spacing of polymers compared to lipids as measured from GISAXS experiments.

The intermediate regions (phase angle $60-70^{\circ}$ ) seem to comprise two parts, orange and light purple, although the boundaries are not clear. The intermediate areas bridge the lipid domains and polymer domains. The direct connections between lipid domains (light yellow) and polymer domains (dark purple) were very rare. These observations suggest that the intermediate regions are the microphase-separated regions composed of mixtures of lipids and polymers, that is, lipid-rich area (orange) and polymer-rich area (light purple). Intriguingly, these microphase-separated regions occupy a larger area than the domains of 
lipid and polymer themselves. This can be attributed to the efforts of the hybrid systems to adjust the large height mismatch between lipid and polymer bilayers.

The area fraction of the purple domains was calculated from the total scan area. For 4:1 molar ratio of lipid to polymer films, the area occupied by the purple and light purple region (polymer-rich) was $27.6 \pm 6 \%$ of the scan area. For 1:1 molar ratio of lipid to polymer films, the area fraction of the polymer domains was $57.2 \pm 7 \%$ and for the 1:4 molar ratio of lipid to polymer films, it was $72.5 \pm 9 \%$. The molecular area of lipid DPPC is $0.65 \mathrm{~nm}^{2},{ }^{68}$ and that of polymer PBD- $b$-PEO is $1.2 \mathrm{~nm}^{2}{ }^{69}$ When the molecular area differences are taken into account, the calculated area of polymer region is $32 \%, 65 \%$, and $88 \%$ for $4: 1,1: 1$, and 1:4 molar ratio of lipid to polymer films, respectively. It should be noted that the area interpretation is qualitative because domain boundaries between lipid and polymer are continuous. Additional AFM images on hybrid films of different lipid to polymer molar ratios are presented in Figure S3 (Supporting Information). Coexistence of macro phaseseparated regions and micro phase-separated regions was also found in hybrid films of different lipid to polymer molar ratios $(4: 1,1: 1,1: 4)$.

To further characterize the region connecting different domains, the cross-section profiles of the phase and height data have been examined as shown in Figure 2D. The phase profile (Figure 2D, top) and corresponding height profile (Figure 2D, bottom) clarify the distinction between lipid/polymer domains and lipid-rich/polymer-rich regions. The lipid and polymer domains remain at a nearly constant height while the intermediate regions show gradual height changes. This supports our interpretation that the intermediate regions (phase angle $60-70^{\circ}$ ) represent an interfacial area where the membrane elastic deformations occur in a gradual way to mitigate line tension at the domain interface. Nonconstant phase values in the interface region also indicate that in this domain, lipid and polymer molecules coexist in a mixed state that extends over a micron meter scale.

AFM phase imaging revealed large phase boundaries between polymers and lipids in the hybrid films. From a materials science perspective, such observations are interesting because phase boundaries are the sites where one often attains unexpected material properties. For example, Yang et al. ${ }^{13}$ recently revealed that the phase boundaries between ordered and disordered lipid domains in plasma membranes are the preferred sites for HIV entry, and the line tension at the phase boundary is a significant driving force of gp41(HIV fusion peptide)mediated fusion. The observations of the unique structures in hybrid films, threedimensional phase separation and relatively large phase boundary area, led us to ask the question of whether membrane-loaded drug molecules would display drug release behavior deviating from that of single-component films. The experiments performed to address this question are described in the next section.

\section{Effect of Paclitaxel Incorporation on the Film Structure.}

We chose Paclitaxel (PTX)-hydrophobic drug to test the release behavior from hybrid films because of the following reasons. First, the local delivery of PTX through thin-film matrices has not been explored with enough detail despite its potential in circumventing the adverse effects of systemic particulate delivery. PTX is known as one of the most effective anticancer drug for the treatment of breast and ovarian cancer. ${ }^{70-72}$ It has been commonly administered 
through long-term systemic injection, which brings unwanted adverse reactions. ${ }^{73}$ The most common adverse effects include infusion-related reactions and neutropenia. ${ }^{73,74}$

Accordingly, local drug delivery to a targeted and focal site of disease (termed surface-or substrate-mediated drug delivery) can be a preferable option for delivering PTX, as in drugeluting medical implants. ${ }^{48,75,76}$ Second, we hypothesized that the unique structure of PTX would maximize the benefit of having domain structures in films. The local delivery of PTX has limited clinical applications due to the crystallization of PTX within the delivery matrix. 77,78 Such crystallization has been the bottleneck in effectively encapsulating PTX into the drug-carrier systems. ${ }^{79}$ When PTX molecules are individually entrapped within the hydrophobic region of bilayers they are in a metastable state ${ }^{80}$ and tend to self-aggregate precipitating out into solution to form a more stable crystalline form. ${ }^{79-83}$

In films composed of single components, the local environment that PTX molecules experience is expected to be similar to the bilayer case described above, that is, there is no effective barrier to hamper the self-aggregation of PTX molecules. CLSM and AFM imaging shown above reveals that the coexistence of polymer-rich and lipid-rich domains in hybrid films generates phase boundaries. Such heterogeneous environment is likely to impose alterations to PTX intermolecular interactions compared to that observed in singlecomponent systems and concomitantly affect PTX crystallization behavior. Indeed, Kan et al. reported that liposomes made of multicomponent lipids that phase-separate into domains exhibited a high encapsulation efficiency of PTX up to $15 \%$ PTX to lipid molar ratio. ${ }^{84}$

We incorporated PTX into the hybrid films and the control films (single-component) varying PTX molar ratios. Figures 3 and 4 summarize the effect of PTX incorporation on the structures of films. Figure 3A shows the AFM data of the hybrid films with PTX where phase color contrast was overlaid onto the peusdo-3D height reconstruction. The whiteyellow domains associated with lipid phase could be clearly differentiated from the purple domains associated with the polymer phase. While the polymer-rich domains (phase angle $55-62^{\circ}$ ) display a rather circular morphology, the shape of lipid-rich domains (phase angle $74-81^{\circ}$ ) is more diverse showing oval and irregular shapes. Additional AFM images of the pure films and hybrid films loaded with PTX can be found in Figure S4.

Figure 3B shows GISAXS I $(q)$ profiles for the hybrid films without PTX (solid black line) and with PTX (gray dotted line). Each profile is composed of two sets of diffraction peaks arising from periodic spacing of polymer-rich and lipid-rich domains. The lamellar peaks associated with polymers are indicated by blue inverted triangles while that of lipids are denoted by red circles. The location of the diffraction peaks was not changed by the addition of PTX molecules, indicating that the lamellar repeat spacing of the hybrid film was maintained, with a periodicity of $a_{\text {lipid }}=57.4 \AA$ and $a_{\text {polymer }}=124 \AA$. Several orders of diffraction peaks (up to ten orders for the polymer phase and up to four orders for the lipid phase) point the high degree of registration in the hybrid films comprising two coexisting phases. We did not observe noticeable differences on the domain size and registration in hybrid films incorporating PTX.

Next, we investigated the in-plane structure of the films by GIWAXS as shown in Figure 4A. Figure 4A displays the 2D X-ray diffraction maps of samples with and without PTX where 
the curvature of the Ewald sphere has taken into account. ${ }^{85,86}$ The pure lipid film (a) shows well-developed in-plane diffraction spots along the $q_{x y}$ axis. These rod-shaped diffraction patterns that manifestly appear in (a) are typical diffraction patterns of two-dimensional lattice systems. Other films containing PTX or polymer (b-f) show broad and less number of scattering patterns compared to (a), reflecting structural transitions toward more fluid-like and short-range ordered molecular arrangements. Note that the intense reflection at $q_{Z} \approx 1.6$ $\AA$ and $q_{x y} \approx 1.2 \AA$ comes from the silicon substrate not from the sample. ${ }^{87}$

In-plane scattering profiles from the 2D data (Figure 4A) were drawn in Figure 4B to obtain the lipid head group and chain correlation spacing (a-f). Multiple peaks in Figure 4B(a) correspond to the diffraction patterns typical of the lipid planar gel phase $\mathrm{L}_{\beta}^{\prime}$ ). It is wellknown that saturated phospholipids such as DPPC form the $\mathrm{L}_{\beta}{ }_{\beta}$ phase below the melting temperature where lipid chains are tilted with respect to the membrane normal and the lipid headgroups and tails form two different $2 \mathrm{D}$ molecular lattices. ${ }^{88-90}$

The Bragg peaks in (a) were best fitted to an orthorhombic lattice for lipid headgroups with lattice parameters $a_{\mathrm{H}}=9.5 \AA$ and $b_{\mathrm{H}}=8.7 \AA\left(\gamma=90^{\circ}\right)$. Lipid tail peaks are hard to be distinguished from the headgroup peaks in (a) but well-packed lipid molecules are known to constitute two different lattices from headgroups and tails. ${ }^{89,91,92}$

Figure $4 \mathrm{~B}(\mathrm{~b})$ shows only one scattering peak at $q=1.5 \AA^{-1}$ in contrast to (a). This implies that the PTX encapsulation inside DPPC films induced a pronounced change in the lipid molecular packing. PTX molecules suppressed long-range order of the lipid headgroups as indicated by the absence of multiple Bragg peaks. The sharp reflection peak at $q \approx 1.5 \AA^{-1}$ comes from the lipid tails packed into a 2-D hexagonal lattice. The q value agrees with the literature references, a characteristic peak of a gel phase $\mathrm{L}_{\beta}$ comprising untilted chains, ${ }^{92,93}$ sometimes referred to a disordered gel phase. ${ }^{94}$ The $\mathrm{L}_{\beta}$ phase has been found in mixtures of DPPC/cholesterol or DPPC/1,2-dioleoyl-sn-glycero-3-phosphocholine (DOPC) lipids. ${ }^{94,95}$

The lipid-polymer hybrid films without PTX (Figure 4B(e)) and with PTX (Figure 4B(f)) present the diffraction peaks of both $\mathrm{L}_{\beta}^{\prime}$ and $\mathrm{L}_{\beta}$ phases coming from lipid tail ordering. Long-range order coming from the lipid head groups was significantly suppressed. Pure polymer films and polymer films loaded with PTX (c, d) did not show well-defined in-plane diffraction patterns because polybutadiene chain blocks are amorphous. For hybrid films, 4:1 lipid to polymer molar ratio is shown because the lipid intensity in hybrid films was significantly decreased compared to that of pure lipid films (further discussed in Figure 4C).

The addition of both molecules (PTX and PBD-PEO) disrupted the tight packing of DPPC molecules, forming a more disordered phase. The presence of PBD-PEO molecules induced a disordering of the DPPC headgroups and tails, however, showed little effect on the changes in molecular tilts compared to PTX molecules which effectively induced a transition to the untilted DPPC gel-phase $\left(\mathrm{L}_{\beta}\right)$.

We further analyzed the scattering curves focusing on the DPPC gel phase peaks. The integrated peak intensity (i.e., area under the peak) was obtained from the background subtracted scattering curves. Figure $4 \mathrm{C}$ (left) shows the integrated peak intensity as a 
function of molar fraction of DPPC in the hybrid films. The red dashed line indicates the case where a decreased amount of the gel phase peak area is in proportion to the decreased molar fraction of DPPC lipids in the hybrid films. If all DPPC lipids remain as the gel phase in the hybrid films, the corresponding gel phase peak area should lie on the red dashed line (e.g., $50 \%$ decrease in the DPPC content should result in 50\% decrease in the integrated peak intensity). However, the calculated gel phase peak area from the hybrid films was only one-fourth of the expected area. Such loss of peak scattering area in the hybrid films is attributed to a large amount of scattering that did not contribute to the gel phase peak scattering. This scattering could be identified as diffuse scattering which appears very broad with no sharp peaks. Such diffuse scattering is indicative of the breakdown of molecular ordering into shorter distances. Various factors such as the rotations of the molecules and reorganization of the acyl chain configurations, or the packing perturbations at the domain boundaries may have accounted for about $75 \%$ loss of the peak scattering.

The observations in the AFM phase image that lipid-rich regions (termed interphases) occupy larger area than lipid domains (Figure 2A) are in a good agreement with the GIWAXS peak intensity analysis where only $\sim 25 \%$ lipids seem to maintain the gel phase and the other $\sim 75 \%$ undergoes a phase transition into a more disordered, fluid-like phase due to the presence of polymer in the stacked membranes. Combining the GIWAXS data (Figure 4C) and AFM data (Figure 2), we deduce that lipid molecules located in the lipid domains far away from the polymer domains are packed in a well-ordered manner over large distances ( $25 \%$ ), while the lipid molecules in the lipid-rich regions mixed with the polymer molecules are in a disordered molecular arrangement $(\sim 75 \%)$. A similar trend was observed for paclitaxel-incorporating films (Figure S5). The loss of the DPPC gel phase peak in the GIWAXS regime was significant for paclitaxel-hybrid films while the lamellar peaks coming from the stacks of DPPC bilayers exhibited intensity signals proportional to DPPC content.

The distribution of PTX drugs within the hybrid films was investigated by CLSM. PTX conjugated with Oregon Green 488 (we termed PTX-Oregon) was used as a fluorophore version of a nonfluorescent molecule PTX. It should be noted that PTX-Oregon has an increased water solubility compared to PTX molecules. ${ }^{96}$ The location of PTX-Oregon in hybrid films can be different from that of pure PTX in hybrid films.

As seen in Figure 5A, we observe a nonhomogeneous distribution of PTX-Oregon in the hybrid films. Green color indicates the location of PTX-Oregon in each of the depthresolved $z$-scan image. The coexistence of lipid and polymer domains with domain boundaries may play a role in the selective partitioning of PTX in the hybrid films. A series of depth-resolved scans revealed that the PTX-incorporated region is not as perfectly aligned along the film normal $(z)$ as seen for domains in hybrid films without PTX (Figure 1A). Such heterogeneous distribution of PTX-Oregon across bilayers could be attributed to the complex domain boundary structures out-of-plane. Higher magnification CLSM images of hybrid films with PTX-Oregon can be found in Figure S6 (Supporting Information).

In Figure 5B, we incorporated $0.1 \mathrm{~mol} \%$ of $\mathrm{DiDC} 18(5)$ to label lipid regions with a dye having different emission and excitation properties to the PTX dye. DiDC18(5), however, did not locate exclusively at the lipid regions unlike NBD-DPPE. When two different 
channels of DiDC18(5) and PTX-Oregon were overlapped (Figure 5B(c)), one could observe non-overlapping regions where solely one color is presented. Such inhomogeneous distribution of two different dye molecules indicates heterogeneous distribution of the lipid and polymer molecules in hybrid films, implying complex interface structures of the hybrid films. Further studies are needed to completely elucidate the preferential location of PTX drugs in the hybrid films. Next, we investigated the PTX release profiles from the films to figure out the potential application of domain structured films in substrate-mediated drug delivery.

\section{Synergistic Release of Paclitaxel from Hybrid Films.}

Figure 6 presents PTX release profiles from supported multilayer films of pure lipid, pure polymer, and lipid-polymer hybrids obtained via RP-HPLC. The films incorporating $2 \mathrm{~mol}$ $\%$ PTX were presented because the PTX loading efficiency was close to $100 \%$ for all films containing 2 mol \% PTX. Therefore, the relative release rates of different film systems could be better compared with respect to domain structure-driven properties. The loading efficiencies for different samples and amount of PTX are summarized in Table S1 (Supporting Information). All drug release profiles showed two sequential stages, an initial fast release followed by a second slower release. The first release stage can be attributed to an initial burst effect ${ }^{98}$ where PTX molecules promptly escape the outer layers of the film surface. Interestingly, the PTX release rate of hybrid films exceeded beyond that of pure lipid films and polymer films, not in between of those two control systems. This occurs without a structural change suggesting a mechanism of membrane permeability enhancement that does not rely on phase transformations. ${ }^{99}$ Figure S7 (Supporting Information) includes the PTX release profiles of different films with varying PTX amount. For the films containing higher molar \% of PTX (5 and $10 \mathrm{~mol} \%$ tested, Figure S7), the same trends (hybrid being the fastest and lipid the slowest) were observed. Although hybrid films comprise a combination of materials that can form single-component films (DPPC or PBD- $b$-PEO), they display a PTX release behavior that is not simply additive. This suggests that structural characteristics of hybrid films are responsible for the observed enhanced transmembrane permeability. Below, we discuss possible connections between phaseseparated domains, domain boundaries, and enhanced transmembrane permeability of hybrid films.

To better understand the mechanism of PTX release kinetics, each of the drug release stages was fitted with various mathematical models, including zero-order kinetics, first-order kinetics, Higuchi model, ${ }^{97}$ Korsmeyer-Peppas model, ${ }^{100,101}$ and Hixson-Crowell model. ${ }^{102}$ On the basis of the correlation coefficient values $R^{2}$, the Higuchi model turned out to be the most suitable model to describe the PTX release from our film systems. The simplified Higuchi model can be described by the following equation $\mathrm{Q}=K^{\mathrm{H}} t^{1 / 2}$ where $\mathrm{Q}$ is the amount of drug released in time $t$, and $K$ is the Higuchi release rate constant. Figure 6A-1 and A-2 show the experimental data plotted as cumulative percentage drug release versus square root of time. The Higuchi model showed good correlations with experimental data as given in Table 1, indicating that drug release from the films was mainly diffusion-controlled with little effect from film swelling or dissolution. 
Our initial hypothesis was that the presence of lipid-polymer domain interfaces would provide an environment able to suppress interactions among PTX molecules, thereby reducing the formation of PTX crystals. To validate such hypothesis, we first checked if the PTX loading efficiency into the film (related to an initial rate of PTX crystal formation) increased for the hybrid films compared to lipid and polymer films. However, as shown in Table S1, there were no noticeable differences in the PTX loading efficiencies between hybrid and pure films. Also, the presence of PTX crystals could not be confirmed from the PTX-containing lipid, polymer, and hybrid films when we carried out GIWAXS experiments on the films under $95 \%$ relative humidity. Only when we prepared aqueous solutions of vesicles of lipid, polymer, and hybrid materials, we could observe the formation of PTX crystals from the WAXS data as shown in Figure 7 and Figure S8 (Supporting Information).

Free PTX molecules in excess water formed needle-like crystals and the diffraction patterns of PTX showed numerous peaks including intense peaks at $8.54^{\circ}, 10.5^{\circ}, 11.1^{\circ}, 12.1^{\circ}, 13.2^{\circ}$ as reported from previous references. ${ }^{103-105}$ When $5 \mathrm{~mol} \%$ PTX is incorporated, lipid and polymer vesicles have some portion of PTX molecules that eventually come out of the bilayer membrane and form crystals. Notably, hybrid samples did not show signs of PTX crystal formation. The reason why we did not observe PTX crystal peaks from the films may originate from the dynamics of PTX crystal formation in films where there is only a humidified atmosphere and not bulk water. The time scale of PTX nucleation, clustering, and aggregation events may be dependent on water content, number of membranes (thickness), among other factors. The detailed mechanism of PTX crystal formation is outside of the scope of the present paper.

To further inspect PTX crystal formation in films, we used optical microscopy (Figure S9 in Supporting Information). Since PTX crystals show characteristic needle-shape, they can be easily distinguished under the microscope. For example, Steffes et al. ${ }^{96}$ investigated the kinetic phase diagrams of PTX solubility in liposomes using differential interference contrast microscopy. Films prepared under the same conditions for PTX release studies were examined. After $16 \mathrm{~h}$ of hydration, PTX crystals were observed from lipid films but not from polymer and hybrid films. Our observation suggests that in hybrid films, there would be simply more PTX molecules available for release. A higher concentration gradient in the hybrid films would lead to an increased PTX permeability. PTX crystal formation is also indirectly reflected in the PTX release profiles where the final release amount did not reach $100 \%$ (Figure S7). The hybrid films released more of the loaded drugs than the pure films. The remaining fraction of the drug could be either precipitated out of the membrane as crystal aggregates or present deep inside the film as small clusters.

Such observations that PTX crystal formation is hampered in lipid-polymer hybrid films raised the next question: why are the hybrid films effective in impeding PTX crystal formation? Kang et al. ${ }^{83}$ demonstrated, via molecular simulations, that PTX molecules in a model membrane display clustering which transform to larger aggregates over time. They also showed that the clustering events depend on the local environment such as the configuration of neighboring PTX molecules within the membrane. On the basis of the simulation study by Kang et al. and our observations, we speculate that hybrid films provide 
a local environment which helps to retain PTX as single molecular states, limiting nucleation and clustering events.

We also consider another possibility contributing to an increased transmembrane permeability: loss of ordering of lipids upon interactions with coexisting polymers in-plane. The inclusion of polymers in the lipid film distorts packing of lipid molecules turning lipid molecular arrangements into more fluid-like and short-range ordering as studied by GIWAXS (Figure 4). The inclusion of polymers in DPPC membranes causes a behavior in the membrane analogous to increasing the temperature ${ }^{106}$ in terms of inducing coexistence of the gel and fluid phases. The interface between gel and fluid phases has been associated with anomalous passive diffusion of ions across membranes due to dynamic fluctuations of the gel and fluid clusters. ${ }^{107-109}$ However, it should be noted that such interfacial regions have much smaller size compared to those of lipid-polymer hybrid films. The concept of leaky interfaces has been also proposed in lipid-polymer systems by means of Monte Carlo simulations. Rabbel et al. ${ }^{110}$ showed that the local permeability of lipid bilayer membranes is strongly increased when a triblock copolymer is incorporated to the bilayer in the transmembrane state. Such increased permeability is attributed to a mismatch in the hydrophobicity between the copolymer middle block and lipid membrane core where a polymer with different hydrophobicity acts as a pore former. We conjecture that increased fluidity of the lipid region upon incorporation of polymers in the membranes would contribute to enhanced transmembrane permeability of PTX through the lipid-polymer hybrid films. It is noteworthy that transport ions through polymer membrane is also enhanced in the presence of membrane domains. ${ }^{111}$

\section{CONCLUSION}

In this work, we have characterized the structure of lipid-polymer hybrid films showing 3D microphase-separation and demonstrated enhanced permeability of hydrophobic drugs incorporated into the hybrid films. Lipid-polymer hybrid films can assemble into multilamellar stacks when supported onto solid substrates. In each lamella, there is lateral microphase-separation of lipids and polymers. The layers are perfectly oriented parallel to the substrate, and the domain alignment across layers is not random. Like-domains stack up as in a smectic phase and the boundaries joining lipid-rich and polymer-rich domains show distinct morphologies. Phase boundaries occupy more area than the domains themselves to alleviate packing frustration arising from the height mismatch between polymer and lipid domains. Interestingly, these microphase separations in 3D serve as functional hotspots for transporting drugs across the bilayers. The hybrid films reveal much faster release rates of hydrophobic drugs (Paclitaxel) compared to single-component films. Plausible mechanisms driving this synergistic release have been suggested in connection with the structure characteristics of the hybrid films. We speculate that two factors contribute to increased permeability of PTX from the hybrid films: (i) an increase in the total concentration of PTX molecules available for release as a result of impeded PTX crystallization and (ii) polymerinduced increase of membrane fluidity.

We have broadened the number of possible applications of lipid films by incorporating block copolymers into the system. Just like in lipid-only systems, in-plane phase separation occurs 
due to local changes in the hydrophobic thickness of films but in a more drastic way. Multistacking of hybrid membranes enables their use for substrate-mediated drug delivery applications as intralayer and interlayer domain correlations are directly impacting the transport of membrane-embedded drugs. Our work also offers future research possibilities on functional lipid-polymer hybrid films beyond drug delivery where synergistic permeation of an embedded solute needs to be controlled.

\section{Supplementary Material}

Refer to Web version on PubMed Central for supplementary material.

\section{ACKNOWLEDGMENTS}

The authors would like to thank Prof. Jianjun Cheng for the generous allowance to use the HPLC equipment. This work was supported by the National Science Foundation under Grant No. DMR-1554435 (Film structure) and the National Institutes of Health under Grant No. 1DP2EB024377-01 (Drug Release). Confocal microscopy imaging experiments were performed at Core Facilities at the Carl R. Woese Institute for Genomic Biology. Synchrotron Xray experiments were carried out at beamline 12-ID-B at the Advanced Photon Source (APS), Argonne National Laboratory. Use of APS was supported by the US Department of Energy (DOE), Office of Science, Office of Basic Energy Sciences, under Contract No. DE-AC02-06CH11357.

\section{REFERENCES}

(1). Singer SJ; Nicolson GL The Fluid Mosaic Model of the Structure of Cell Membranes. Science 1972, 175, 720-731. [PubMed: 4333397]

(2). Estep TN; Mountcastle DB; Barenholz Y; Biltonen RL; Thompson TE Thermal Behavior of Synthetic Sphingomyelin-Cholesterol Dispersions. Biochemistry 1979, 18, 2112-2117. [PubMed: 435470]

(3). Goodsaid-Zalduondo F; Rintoul DA; Carlson JC; Hansel W Luteolysis-Induced Changes in Phase Composition and Fluidity of Bovine Luteal Cell Membranes. Proc. Natl. Acad. Sci. U. S. A 1982, 79, 4332-4336. [PubMed: 6956862]

(4). Simons K; Van Meer G Lipid Sorting in Epithelial Cells. Biochemistry 1988, 27, 6197-6202. [PubMed: 3064805]

(5). Simons K; Ikonen E Functional Rafts in Cell Membranes. Nature 1997, 387, 569-572. [PubMed: 9177342]

(6). Pike LJ The Challenge of Lipid Rafts. J. Lipid Res 2009, 50, S323-S328. [PubMed: 18955730]

(7). Martens JR; Navarro-Polanco R; Coppock EA; Nishiyama A; Parshley L; Grobaski TD; Tamkun MM Differential Targeting of Shaker-like Potassium Channels to Lipid Rafts. J. Biol. Chem 2000, 275, 7443-7446. [PubMed: 10713042]

(8). Yarbrough TL; Lu T; Lee H-C; Shibata EF Localization of Cardiac Sodium Channels in CaveolinRich Membrane Domains: Regulation of Sodium Current Amplitude. Circ. Res 2002, 90, 443 449. [PubMed: 11884374]

(9). Darby PJ; Kwan CY; Daniel EE Caveolae from Canine Airway Smooth Muscle Contain the Necessary Components for a Role in $\mathrm{Ca}(2+)$ Handling. Am. J. Physiol. Lung Cell. Mol. Physiol 2000, 279, L1226-L1235. [PubMed: 11076813]

(10). Owen DM; Williamson DJ; Magenau A; Gaus K Sub-Resolution Lipid Domains Exist in the Plasma Membrane and Regulate Protein Diffusion and Distribution. Nat. Commun 2012, 3, 1256. [PubMed: 23212385]

(11). Chazal N; Gerlier D Virus Entry, Assembly, Budding, and Membrane Rafts. Microbiol. Mol. Biol. Rev. MMBR 2003, 67, 226-237. [PubMed: 12794191]

(12). Pietiäinen VM; Marjomäki V; Heino J; Hyypiä T Viral Entry, Lipid Rafts and Caveosomes. Ann. Med 2005, 37, 394-403. [PubMed: 16203612] 
(13). Yang S-T; Kiessling V; Tamm LK Line Tension at Lipid Phase Boundaries as Driving Force for HIV Fusion Peptide-Mediated Fusion. Nat. Commun 2016, 7, 11401. [PubMed: 27113279]

(14). Binder WH; Barragan V; Menger FM Domains and Rafts in Lipid Membranes. Angew. Chem., Int. Ed 2003, 42, 5802-5827.

(15). Olubummo A; Schulz M; Lechner B-D; Scholtysek P; Bacia K; Blume A; Kressler J; Binder WH Controlling the Localization of Polymer-Functionalized Nanoparticles in Mixed Lipid/Polymer Membranes. ACS Nano 2012, 6, 8713-8727. [PubMed: 22950802]

(16). Kowal J; Wu D; Mikhalevich V; Palivan CG; Meier W Hybrid Polymer-Lipid Films as Platforms for Directed Membrane Protein Insertion. Langmuir 2015, 31, 4868-4877. [PubMed: 25849126]

(17). Heath GR; Li M; Rong H; Radu V; Frielingsdorf S; Lenz O; Butt JN; Jeuken LJC Multilayered Lipid Membrane Stacks for Biocatalysis Using Membrane Enzymes. Adv. Funct. Mater 2017, 27, 1606265.

(18). Kang M; Leal C Soft Nanostructured Films for Actuated Surface-Based siRNA Delivery. Adv. Funct. Mater 2016, 26, 5610-5620.

(19). Kang M; Kim H; Leal C Self-Organization of Nucleic Acids in Lipid Constructs. Curr. Opin. Colloid Interface Sci 2016, 26, 58-65. [PubMed: 28496379]

(20). Lee CH; Kim H; Harburg DV; Park G; Ma Y; Pan T; Kim JS; Lee NY; Kim BH; Jang K-I; et al. Biological Lipid Membranes for on-Demand, Wireless Drug Delivery from Thin, Bioresorbable Electronic Implants. NPG Asia Mater. 2015, 7, e227. [PubMed: 27175221]

(21). Steer D; Kang M; Leal C Soft Nanostructured Films for Directing the Assembly of Functional Materials. Nanotechnology 2017, 28, 142001. [PubMed: 28145900]

(22). Liu Y; Liu B; Nie Z Concurrent Self-Assembly of Amphiphiles into Nanoarchitectures with Increasing Complexity. Nano Today 2015, 10, 278-300.

(23). Schulz M; Binder WH Mixed Hybrid Lipid/Polymer Vesicles as a Novel Membrane Platform. Macromol. Rapid Commun 2015, 36, 2031-2041. [PubMed: 26457675]

(24). Chen C; Wylie RAL; Klinger D; Connal LA Shape Control of Soft Nanoparticles and Their Assemblies. Chem. Mater 2017, 29, 1918-1945.

(25). Nam J; Beales PA; Vanderlick TK Giant Phospholipid/Block Copolymer Hybrid Vesicles: Mixing Behavior and Domain Formation. Langmuir 2011, 27, 1-6. [PubMed: 21133340]

(26). Schulz M; Glatte D; Meister A; Scholtysek P; Kerth A; Blume A; Bacia K; Binder WH Hybrid Lipid/Polymer Giant Unilamellar Vesicles: Effects of Incorporated Biocompatible PIB-PEO Block Copolymers on Vesicle Properties. Soft Matter 2011, 7, 8100-8110.

(27). Nam J; Vanderlick TK; Beales PA Formation and Dissolution of Phospholipid Domains with Varying Textures in Hybrid Lipo-Polymersomes. Soft Matter 2012, 8, 7982-7988.

(28). Schulz M; Olubummo A; Bacia K; Binder WH Lateral Surface Engineering of Hybrid lipid-BCP Vesicles and Selective Nanoparticle Embedding. Soft Matter 2014, 10, 831-839. [PubMed: 24837370]

(29). Chen D; Santore MM Hybrid Copolymer-phospholipid Vesicles: Phase Separation Resembling Mixed Phospholipid Lamellae, but with Mechanical Stability and Control. Soft Matter 2015, 11, 2617-2626. [PubMed: 25687473]

(30). Dao TPT; Fernandes F; Ibarboure E; Ferji K; Prieto M; Sandre O; Le Meins J-F Modulation of Phase Separation at the Micron Scale and Nanoscale in Giant Polymer/Lipid Hybrid Unilamellar Vesicles (GHUVs). Soft Matter 2017, 13, 627-637. [PubMed: 27991638]

(31). Dao TPT; Brûlet A; Fernandes F; Er-Rafik M; Ferji K; Schweins R; Chapel J-P; Fedorov A; Schmutz M; Prieto M; et al. Mixing Block Copolymers with Phospholipids at the Nanoscale: From Hybrid Polymer/Lipid Wormlike Micelles to Vesicles Presenting Lipid Nanodomains. Langmuir 2017, 33, 1705-1715. [PubMed: 28128560]

(32). Gettel DL; Sanborn J; Patel MA; de Hoog H-P; Liedberg B; Nallani M; Parikh AN Mixing, Diffusion, and Percolation in Binary Supported Membranes Containing Mixtures of Lipids and Amphiphilic Block Copolymers. J. Am. Chem. Soc 2014, 136, 10186-10189. [PubMed: 25003585]

(33). Itel F; Najer A; Palivan CG; Meier W Dynamics of Membrane Proteins within Synthetic Polymer Membranes with Large Hydrophobic Mismatch. Nano Lett. 2015, 15, 3871-3878. [PubMed: 26013972] 
(34). LoPresti C; Massignani M; Fernyhough C; Blanazs A; Ryan AJ; Madsen J; Warren NJ; Armes SP; Lewis AL; Chirasatitsin S; et al. Controlling Polymersome Surface Topology at the Nanoscale by Membrane Confined Polymer/Polymer Phase Separation. ACS Nano 2011, 5, 1775-1784. [PubMed: 21344879]

(35). Le Meins J-F; Schatz C; Lecommandoux S; Sandre O Hybrid Polymer/Lipid Vesicles: State of the Art and Future Perspectives. Mater. Today 2013, 16, 397-402.

(36). Schulz M; Olubummo A; Binder WH Beyond the Lipid-Bilayer: Interaction of Polymers and Nanoparticles with Membranes. Soft Matter 2012, 8, 4849-4864.

(37). Hinman SS; Ruiz CJ; Cao Y; Ma MC; Tang J; Laurini E; Posocco P; Giorgio S; Pricl S; Peng L; et al. Mix and Match: Coassembly of Amphiphilic Dendrimers and Phospholipids Creates Robust, Modular, and Controllable Interfaces. ACS Appl. Mater. Interfaces 2017, 9, 1029-1035. [PubMed: 27957833]

(38). Sasaki DY; Stevens MJ Stacked, Folded, and Bent Lipid Membranes. MRS Bull. 2006, 31, 521526.

(39). McMahon HT; Gallop JL Membrane Curvature and Mechanisms of Dynamic Cell Membrane Remodelling. Nature 2005, 438, 590-596. [PubMed: 16319878]

(40). Anderson JM Lateral Heterogeneity of Plant Thylakoid Protein Complexes: Early Reminiscences. Philos. Trans. R. Soc., B 2012, 367, 3384-3388.

(41). Pribil M; Labs M; Leister D Structure and Dynamics of Thylakoids in Land Plants. J. Exp. Bot 2014, 65, 1955-1972. [PubMed: 24622954]

(42). Kirchhoff H; Sharpe RM; Herbstova M; Yarbrough R; Edwards GE Differential Mobility of Pigment-Protein Complexes in Granal and Agranal Thylakoid Membranes of C3 and C4 Plants. Plant Physiol. 2013, 161, 497-507. [PubMed: 23148078]

(43). Zelikin AN Drug Releasing Polymer Thin Films: New Era of Surface-Mediated Drug Delivery. ACS Nano 2010, 4, 2494-2509. [PubMed: 20423067]

(44). Kearney CJ; Mooney DJ Macroscale Delivery Systems for Molecular and Cellular Payloads. Nat. Mater 2013, 12, 1004-1017. [PubMed: 24150418]

(45). Wang Y; Angelatos AS; Caruso F Template Synthesis of Nanostructured Materials via Layer-byLayer Assembly. Chem. Mater 2008, 20, 848-858.

(46). Alexis F; Venkatraman SS; Rath SK; Boey F In Vitro Study of Release Mechanisms of Paclitaxel and Rapamycin from Drug-Incorporated Biodegradable Stent Matrices. J. Controlled Release 2004, 98, 67-74.

(47). Yamauchi F; Koyamatsu Y; Kato K; Iwata H Layer-by-Layer Assembly of Cationic Lipid and Plasmid DNA onto Gold Surface for Stent-Assisted Gene Transfer. Biomaterials 2006, 27, $3497-$ 3504. [PubMed: 16488471]

(48). Zilberman M; Kraitzer A; Grinberg O; Elsner JJ Drug-Eluting Medical Implants. Handb. Exp. Pharmacol 2010, 197, 299-341.

(49). Gribova V; Auzely-Velty R; Picart C Polyelectrolyte Multilayer Assemblies on Materials Surfaces: From Cell Adhesion to Tissue Engineering. Chem. Mater 2012, 24, 854-869. [PubMed: 25076811]

(50). Chassepot A; Gao L; Nguyen I; Dochter A; Fioretti F; Menu P; Kerdjoudj H; Baehr C; Schaaf P; Voegel J-C; et al. Chemically Detachable Polyelectrolyte Multilayer Platform for Cell Sheet Engineering. Chem. Mater 2012, 24, 930-937.

(51). Chen X; Cortez-Jugo C; Choi GH; Björnmalm M; Dai Y; Yoo PJ; Caruso F Patterned Poly(dopamine) Films for Enhanced Cell Adhesion. Bioconjugate Chem. 2017, 28, 75-80.

(52). Tayebi L; Ma Y; Vashaee D; Chen G; Sinha SK; Parikh AN Long-Range Interlayer Alignment of Intralayer Domains in Stacked Lipid Bilayers. Nat. Mater 2012, 11, 1074-1080. [PubMed: 23085566]

(53). Ilavsky J; Jemian PR Irena: Tool Suite for Modeling and Analysis of Small-Angle Scattering. J. Appl. Crystallogr 2009, 42, 347-353.

(54). Hong Y; Ye S-H; Pelinescu AL; Wagner WR Synthesis, Characterization, and Paclitaxel Release from a Biodegradable, Elastomeric, Poly(ester Urethane)urea Bearing Phosphorylcholine Groups for Reduced Thrombogenicity. Biomacromolecules 2012, 13, 3686-3694. [PubMed: 23035885] 
(55). Goldspiel BR Clinical Overview of the Taxanes. Pharmacotherapy 1997, 17, 110S-125S. [PubMed: 9322878]

(56). Crane JM; Tamm LK Role of Cholesterol in the Formation and Nature of Lipid Rafts in Planar and Spherical Model Membranes. Biophys. J 2004, 86, 2965-2979. [PubMed: 15111412]

(57). Björkqvist YJE; Nyholm TKM; Slotte JP; Ramstedt B Domain Formation and Stability in Complex Lipid Bilayers as Reported by Cholestatrienol. Biophys. J 2005, 88, 4054-4063. [PubMed: 15792981]

(58). Pathak P; London E The Effect of Membrane Lipid Composition on the Formation of Lipid Ultrananodomains. Biophys. J 2015, 109, 1630-1638. [PubMed: 26488654]

(59). Bagatolli LA; Gratton E A Correlation between Lipid Domain Shape and Binary Phospholipid Mixture Composition in Free Standing Bilayers: A Two-Photon Fluorescence Microscopy Study. Biophys. J 2000, 79, 434-447. [PubMed: 10866969]

(60). Helfrich W Steric Interaction of Fluid Membranes in Multilayer Systems. Z. Naturforsch., A: Phys. Sci 1978, 33, 305-315.

(61). Evans EA; Parsegian VA Thermal-Mechanical Fluctuations Enhance Repulsion between Bimolecular Layers. Proc. Natl. Acad. Sci. U. S. A 1986, 83, 7132-7136. [PubMed: 3463955]

(62). Milhaud J New Insights into Water-phospholipid Model Membrane Interactions. Biochim. Biophys. Acta, Biomembr 2004, 1663, 19-51.

(63). Kuzmin PI; Akimov SA; Chizmadzhev YA; Zimmerberg J; Cohen FS Line Tension and Interaction Energies of Membrane Rafts Calculated from Lipid Splay and Tilt. Biophys. J 2005, 88, 1120-1133. [PubMed: 15542550]

(64). Nagle JF; Wilkinson DA Dilatometric Studies of the Subtransition in Dipalmitoylphosphatidylcholine. Biochemistry 1982, 21, 3817-3821. [PubMed: 6897192]

(65). Tristram-Nagle S; Nagle JF Lipid Bilayers: Thermodynamics, Structure, Fluctuations, and Interactions. Chem. Phys. Lipids 2004, 127, 3-14. [PubMed: 14706737]

(66). Pang GKH; Baba-Kishi KZ; Patel A Topographic and Phase-Contrast Imaging in Atomic Force Microscopy. Ultramicroscopy 2000, 81, 35-40. [PubMed: 10998788]

(67). Raghavan D; VanLandingham M; Gu X; Nguyen T Characterization of Heterogeneous Regions in Polymer Systems Using Tapping Mode and Force Mode Atomic Force Microscopy. Langmuir 2000, 16, 9448-9459.

(68). Leekumjorn S; Sum AK Molecular Simulation Study of Structural and Dynamic Properties of Mixed DPPC/DPPE Bilayers. Biophys. J 2006, 90, 3951-3965. [PubMed: 16533838]

(69). He F; Tong YW A Mechanistic Study on Amphiphilic Block Co-Polymer Poly(butadiene-B(Ethylene Oxide)) Vesicles Reveals the Water Permeation Mechanism through a Polymeric Bilayer. RSC Adv. 2014, 4, 15304-15313.

(70). Wiseman LR; Spencer CM Paclitaxel. An Update of Its Use in the Treatment of Metastatic Breast Cancer and Ovarian and Other Gynaecological Cancers. Drugs Aging 1998, 12, 305-334. [PubMed: 9571394]

(71). Rowinsky EK; Onetto N; Canetta RM; Arbuck SG Taxol: The First of the Taxanes, an Important New Class of Antitumor Agents. Semin. Oncol 1992, 19, 646-662. [PubMed: 1361079]

(72). Slichenmyer WJ; Von Hoff DD Taxol: A New and Effective Anti-Cancer Drug. Anti-Cancer Drugs 1991, 2, 519-530. [PubMed: 1687206]

(73). Scripture C; Figg W; Sparreboom A Peripheral Neuropathy Induced by Paclitaxel: Recent Insights and Future Perspectives. Curr. Neuropharmacol 2006, 4, 165-172. [PubMed: 18615126]

(74). Lehoczky O; Bagaméri A; Udvary J; Pulay T Side-Effects of Paclitaxel Therapy in Ovarian Cancer Patents. Eur. J. Gynaecol. Oncol 2001, 22, 81-84. [PubMed: 11321504]

(75). Bertrand OF; Sipehia R; Mongrain R; Rodes J; Tardif JC; Bilodeau L; Côté G; Bourassa MG Biocompatibility Aspects of New Stent Technology. J. Am. Coll. Cardiol 1998, 32, 562-571. [PubMed: 9741494]

(76). Nakazawa G; Finn AV; Ladich E; Ribichini F; Coleman L; Kolodgie FD; Virmani R DrugEluting Stent Safety: Findings from Preclinical Studies. Expert Rev. Cardiovasc. Ther 2008, 6, 1379-1391. [PubMed: 19018691] 
(77). S. J; V. Tapia L; A R; A C; A P; Ozdemir O Negative Impact of Paclitaxel Crystallization on Hydrogels and Novel Approaches for Anticancer Drug Delivery Systems. Current Cancer Treatment - Novel Beyond Conventional Approaches 2011, 767.

(78). Castro JS; Deymier PA; Trzaskowski B; Bucay J Heterogeneous and Homogeneous Nucleation of Taxol Crystals in Aqueous Solutions and Gels: Effect of Tubulin Proteins. Colloids Surf., B 2010, 76, 199-206.

(79). Koudelka Š; Turének J Liposomal Paclitaxel Formulations. J. Controlled Release 2012, 163, 322334.

(80). Wenk MR; Fahr A; Reszka R; Seelig J Paclitaxel Partitioning into Lipid Bilayers. J. Pharm. Sci 1996, 85, 228-231. [PubMed: 8683453]

(81). Balasubramanian SV; Alderfer JL; Straubinger RM Solvent- and Concentration-Dependent Molecular Interactions of Taxol (Paclitaxel †). J. Pharm. Sci 1994, 83, 1470-1476. [PubMed: 7884672]

(82). Mastropaolo D; Camerman A; Luo Y; Brayer GD; Camerman N Crystal and Molecular Structure of Paclitaxel (Taxol). Proc. Natl. Acad. Sci. U. S. A 1995, 92, 6920-6924. [PubMed: 7624344]

(83). Kang M; Loverde SM Molecular Simulation of the Concentration-Dependent Interaction of Hydrophobic Drugs with Model Cellular Membranes. J. Phys. Chem. B 2014, 118, 11965 11972. [PubMed: 25243601]

(84). Kan P; Tsao C-W; Wang A-J; Su W-C; Liang H-F A Liposomal Formulation Able to Incorporate a High Content of Paclitaxel and Exert Promising Anticancer Effect. J. Drug Delivery 2011, 2011, 1-9.

(85). Breiby DW; Bunk O; Andreasen JW; Lemke HT; Nielsen MM Simulating X-Ray Diffraction of Textured Films. J. Appl. Crystallogr 2008, 41, 262-271.

(86). Baker JL; Jimison LH; Mannsfeld S; Volkman S; Yin S; Subramanian V; Salleo A; Alivisatos AP; Toney MF Quantification of Thin Film Crystallographic Orientation Using XRay Diffraction with an Area Detector. Langmuir 2010, 26, 9146-9151. [PubMed: 20361783]

(87). Weinhausen B; Aeffner S; Reusch T; Salditt T Acyl-Chain Correlation in Membrane Fusion Intermediates: X-Ray Diffraction from the Rhombohedral Lipid Phase. Biophys. J 2012, 102, 2121-2129. [PubMed: 22824276]

(88). Sun W-J; Suter RM; Knewtson MA; Worthington CR; Tristram-Nagle S; Zhang R; Nagle JF Order and Disorder in Fully Hydrated Unoriented Bilayers of Gel-Phase Dipalmitoylphosphatidylcholine. Phys. Rev. E: Stat. Phys., Plasmas, Fluids, Relat. Interdiscip. Top 1994, 49, 4665-4676.

(89). Barrett MA; Zheng S; Roshankar G; Alsop RJ; Belanger RKR; Huynh C; Kučerka N; Rheinstädter MC Interaction of Aspirin (Acetylsalicylic Acid) with Lipid Membranes. PLoS One 2012, 7, e34357. [PubMed: 22529913]

(90). Schubert T; Schneck E; Tanaka M First Order Melting Transitions of Highly Ordered Dipalmitoyl Phosphatidylcholine Gel Phase Membranes in Molecular Dynamics Simulations with Atomistic Detail. J. Chem. Phys 2011, 135, 055105. [PubMed: 21823736]

(91). Katsaras J; Raghunathan VA; Dufourc EJ; Dufourcq J Evidence for a Two-Dimensional Molecular Lattice in Subgel Phase DPPC Bilayers. Biochemistry 1995, 34, 4684-4688. [PubMed: 7718572]

(92). Armstrong CL; Marquardt D; Dies H; Kučerka N; Yamani Z; Harroun TA; Katsaras J; Shi A-C; Rheinstädter MC The Observation of Highly Ordered Domains in Membranes with Cholesterol. PLoS One 2013, 8, e66162. [PubMed: 23823623]

(93). Alsop RJ; Armstrong CL; Maqbool A; Toppozini L; Dies H; Rheinstädter MC Cholesterol Expels Ibuprofen from the Hydrophobic Membrane Core and Stabilizes Lamellar Phases in Lipid Membranes Containing Ibuprofen. Soft Matter 2015, 11, 4756-4767. [PubMed: 25915907]

(94). Mills TT; Huang J; Feigenson GW; Nagle JF Effects of Cholesterol and Unsaturated DOPC Lipid on Chain Packing of Saturated Gel-Phase DPPC Bilayers. Gen. Physiol. Biophys 2009, 28, 126139. [PubMed: 19592709]

(95). Clarke JA; Heron AJ; Seddon JM; Law RV The Diversity of the Liquid Ordered (Lo) Phase of Phosphatidylcholine/Cholesterol Membranes: A Variable Temperature Multinuclear Solid-State NMR and X-Ray Diffraction Study. Biophys. J 2006, 90, 2383-2393. [PubMed: 16537550] 
(96). Steffes VM; Murali MM; Park Y; Fletcher BJ; Ewert KK; Safinya CR Distinct Solubility and Cytotoxicity Regimes of Paclitaxel-Loaded Cationic Liposomes at Low and High Drug Content Revealed by Kinetic Phase Behavior and Cancer Cell Viability Studies. Biomaterials 2017, 145, 242-255. [PubMed: 28889081]

(97). Higuchi T Mechanism of Sustained-action Medication. Theoretical Analysis of Rate of Release of Solid Drugs Dispersed in Solid Matrices. J. Pharm. Sci 1963, 52, 1145-1149. [PubMed: 14088963]

(98). Huang X; Brazel CS On the Importance and Mechanisms of Burst Release in Matrix-Controlled Drug Delivery Systems. J. Controlled Release 2001, 73, 121-136.

(99). Kang M; Huang G; Leal C Role of Lipid Polymorphism in Acoustically Sensitive Liposomes. Soft Matter 2014, 10, 8846-8854. [PubMed: 25286018]

(100). Korsmeyer RW; Gurny R; Doelker E; Buri P; Peppas NA Mechanisms of Solute Release from Porous Hydrophilic Polymers. Int. J. Pharm 1983, 15, 25-35.

(101). Peppas NA A Model of Dissolution-Controlled Solute Release from Porous Drug Delivery Polymeric Systems. J. Biomed. Mater. Res 1983, 17, 1079-1087. [PubMed: 6654927]

(102). Hixson AW; Crowell JH Dependence of Reaction Velocity upon Surface and Agitation. Ind. Eng. Chem 1931, 23, 923-931.

(103). Zhang D; Wang. Folate-Mediated Targeted and Intracellular Delivery of Paclitaxel Using a Novel Deoxycholic Acid-O-Carboxymethylated Chitosan and Folic Acid Micelles. Int. J. Nanomed 2012, 7, 325-337.

(104). Zhang H; Hu H; Zhang H; Dai W; Wang X; Wang X; Zhang Q Effects of PEGylated Paclitaxel Nanocrystals on Breast Cancer and Its Lung Metastasis. Nanoscale 2015, 7, 10790-10800. [PubMed: 26038337]

(105). Zhong T; Yao X; Zhang S; Guo Y; Duan X-C; Ren W; Huang D; Yin Y-F; Zhang X A SelfAssembling Nanomedicine of Conjugated Linoleic Acid-Paclitaxel Conjugate (CLA-PTX) with Higher Drug Loading and Carrier-Free Characteristic. Sci. Rep 2016, 6, 36614. [PubMed: 27812039]

(106). Leekumjorn S; Sum AK Molecular Studies of the Gel to Liquid-Crystalline Phase Transition for Fully Hydrated DPPC and DPPE Bilayers. Biochim. Biophys. Acta, Biomembr 2007, 1768, 354365.

(107). Cruzeiro-Hansson L; Mouritsen OG Passive Ion Permeability of Lipid Membranes Modelled via Lipid-Domain Interfacial Area. Biochim. Biophys. Acta, Biomembr 1988, 944, 63-72.

(108). Zhernenkov M; Bolmatov D; Soloviov D; Zhernenkov K; Toperverg BP; Cunsolo A; Bosak A; Cai YQ Revealing the Mechanism of Passive Transport in Lipid Bilayers via Phonon-Mediated Nanometre-Scale Density Fluctuations. Nat. Commun 2016, 7, 11575. [PubMed: 27175859]

(109). Risbo J; Jørgensen K; Sperotto MM; Mouritsen OG Phase Behavior and Permeability Properties of Phospholipid Bilayers Containing a Short-Chain Phospholipid Permeability Enhancer. Biochim. Biophys. Acta, Biomembr 1997, 1329, 85-96.

(110). Rabbel H; Werner M; Sommer J-U Interactions of Amphiphilic Triblock Copolymers with Lipid Membranes: Modes of Interaction and Effect on Permeability Examined by Generic Monte Carlo Simulations. Macromolecules 2015, 48, 4724-4732.

(111). Evans CM; Sanoja GE; Popere BC; Segalman RA Anhydrous Proton Transport in Polymerized Ionic Liquid Block Copolymers: Roles of Block Length, Ionic Content, and Confinement. Macromolecules 2016, 49, 395-404. 

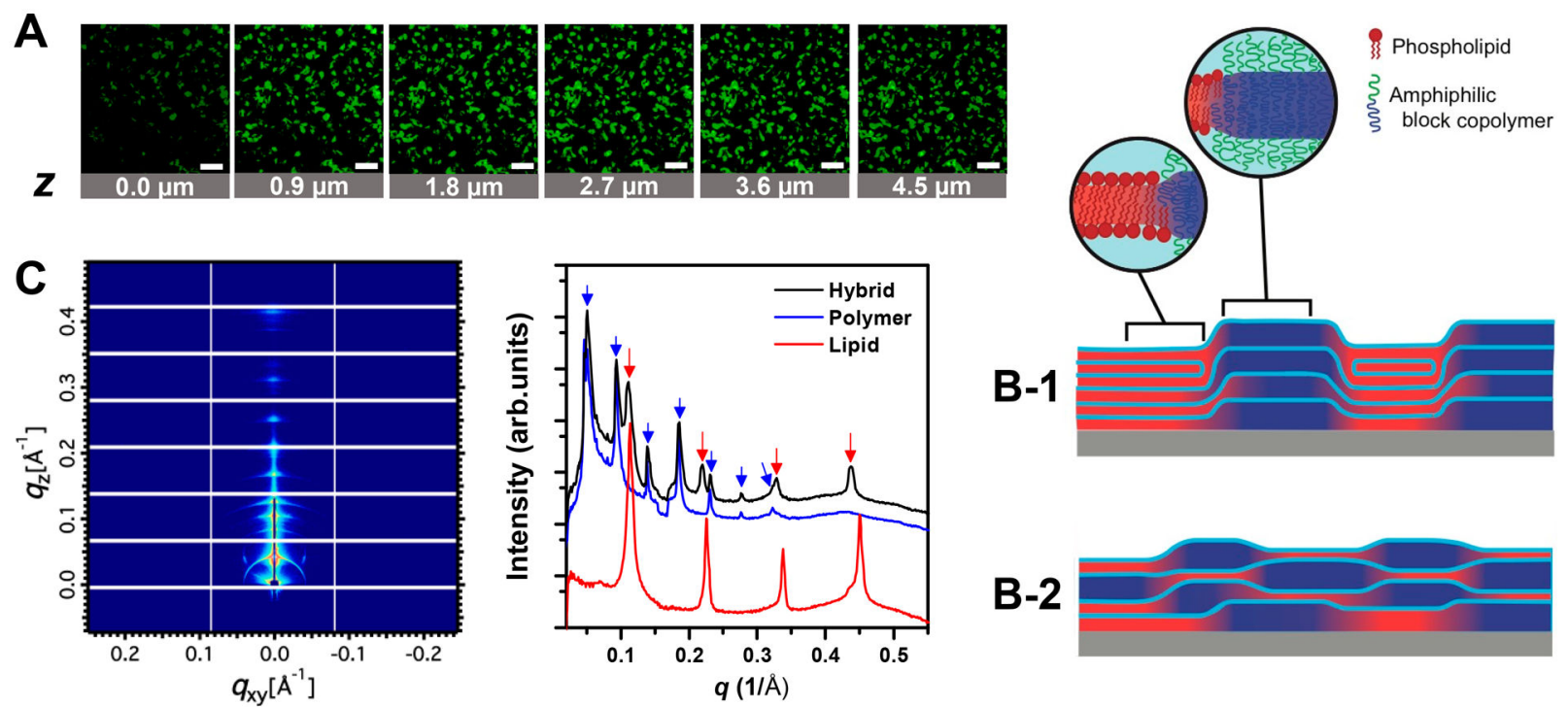

Figure 1.

Structural characterization of lipid-polymer hybrid films (1:1 molar ratio of DPPC to PBD$b$-PEO). (A) Confocal laser scanning microscopy images of hybrid films in bulk water doped with NBD-DPPE $(0.1 \mathrm{~mol} \%)$. A series of images with height variation was taken to examine the phase separation in hybrid films at different depths. Binary spatial patterns continue across the bilayer membrane normal, implying the alignment of domains across multilamellar films. Scale bars $=50 \mu \mathrm{m}$. (B) Schematic illustration of hybrid films consisting of lipid (red) domains and polymer (dark blue) domains. (B-1) Case when the like-domains align on top of each other across layers. (B-2) Random alignment of domains in neighboring layers. Each block represents a single bilayer of lipid or polymer, and the spaces between domains (sky blue) represent water layers. (C) GISAXS two-dimensional raw data of hybrid films (left) and one-dimensional $\mathrm{I}(q)$ profiles of hybrid, lipid, and polymer films (right) at $>95 \%$ relative humidity. The diffraction peaks from hybrid films comprise the sum of diffraction peaks from lipid and polymer films. 

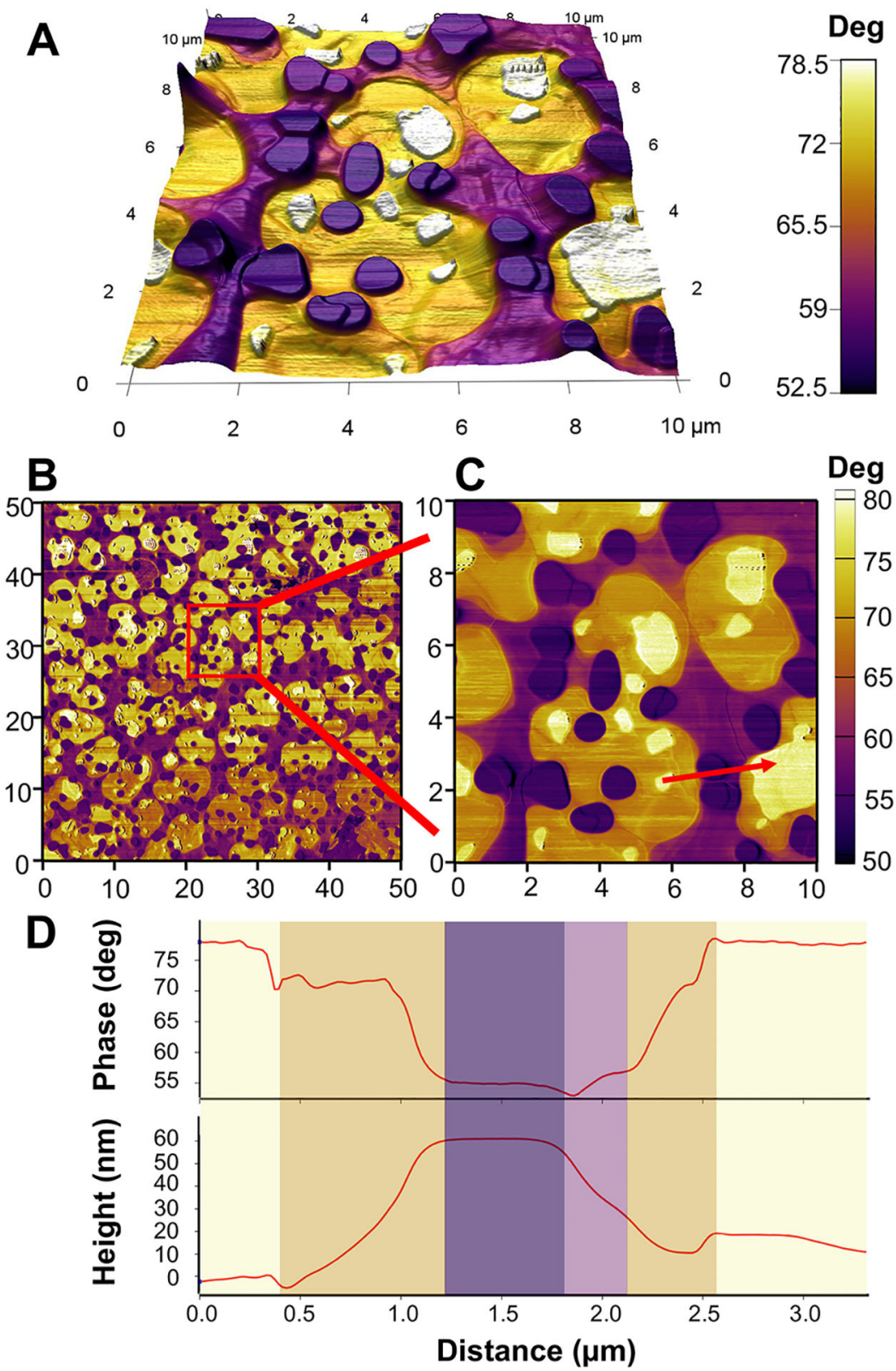

Figure 2.

Surface characterization of hybrid films using topographic and phase-contrast imaging in AFM. (A) Phase contrast image overlaid onto pseudo-3D topography of hybrid films (4:1 molar ratio of DPPC to PBD- $b$-PEO). Note the high color contrast between different regions, indicative of the presence of different domains. Field of view $=10 \mu \mathrm{m} \times 10 \mu \mathrm{m}$. (B, C) Phase contrast AFM images of hybrid films (4:1 molar ratio of DPPC to PBD- $b$-PEO) showing well-defined phase-separated domains. The units on the $x$-and $y$-axis in the plots are $\mu \mathrm{m}$. (D) Cross-sectional profiles of the phase (top) and topography (bottom) along the arrow marked in panel $\mathrm{C}$. The background color reflects the corresponding phase contrast of each region. 

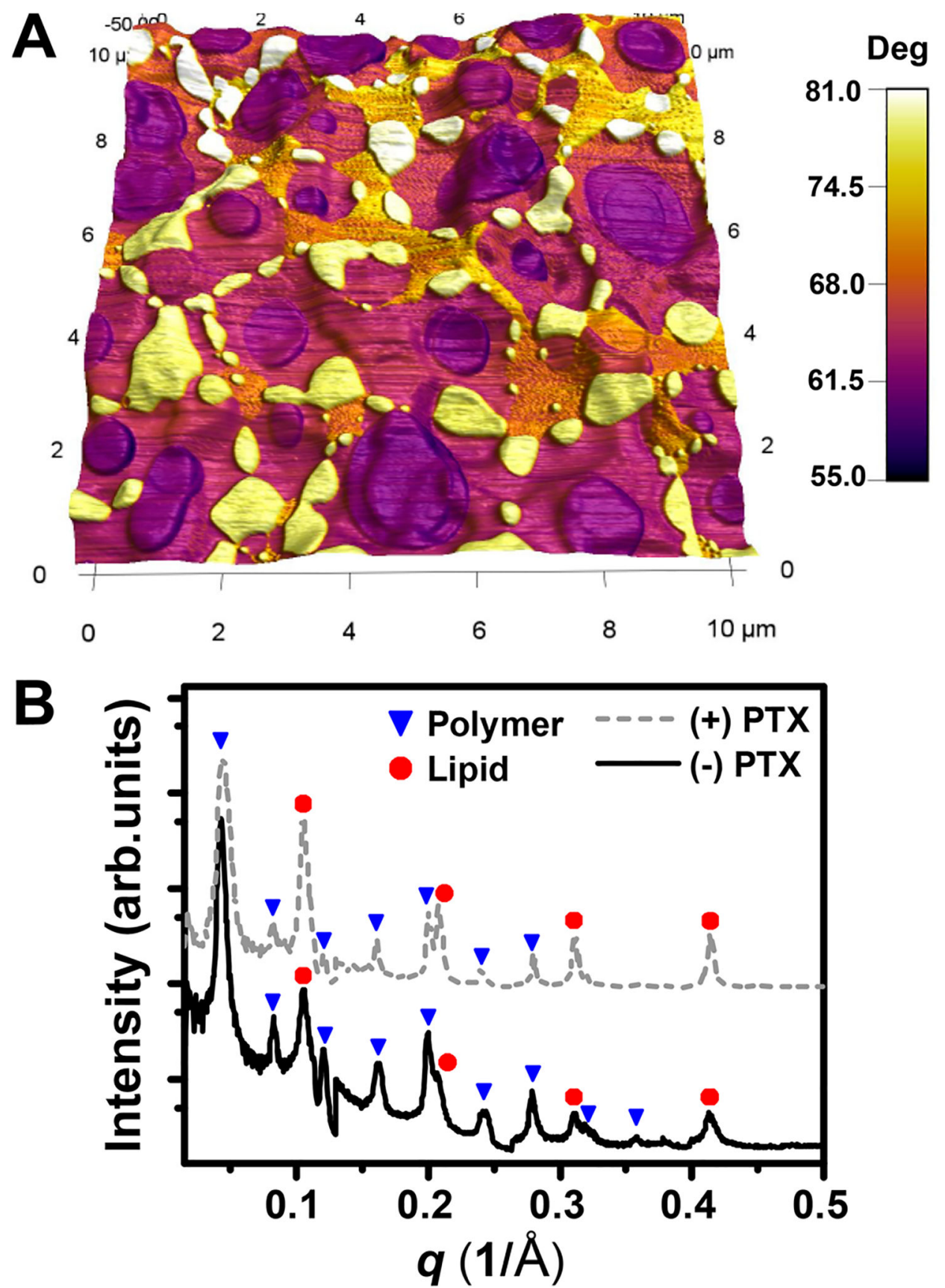

Figure 3.

(A) Phase contrast image overlaid onto pseudo-3D topography of drug-incorporated hybrid films (1:1 molar ratio of DPPC to PBD- $b$-PEO $+5 \mathrm{~mol} \%$ PTX). Field of view $=10 \mu \mathrm{m} \times 10$ $\mu \mathrm{m}$. (B) GISAXS one-dimensional I $(q)$ profiles of $1: 1$ molar ratio of DPPC to PBD- $b$-PEO hybrid films before and after 5 mol \% PTX incorporation. 
A
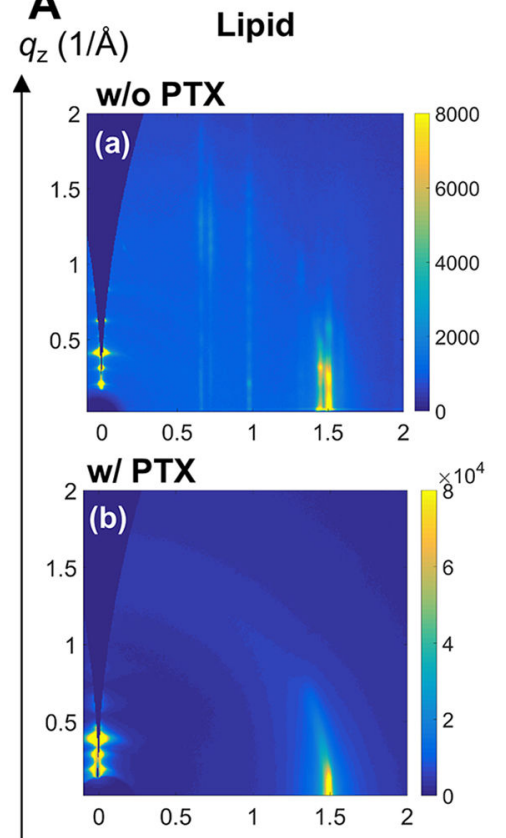

Polymer
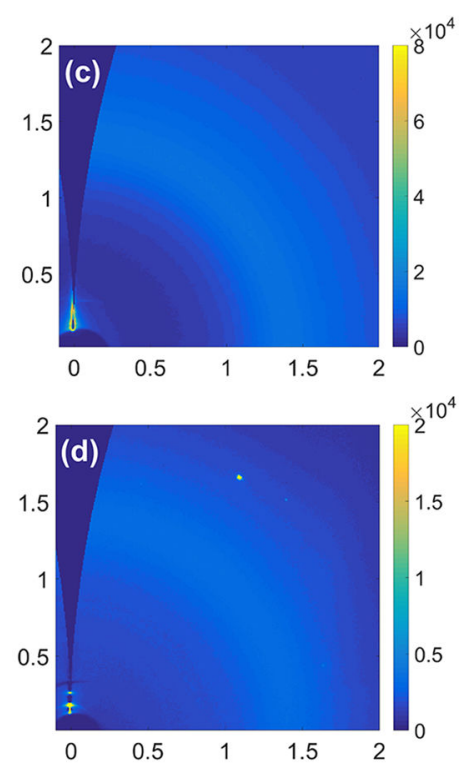

Hybrid
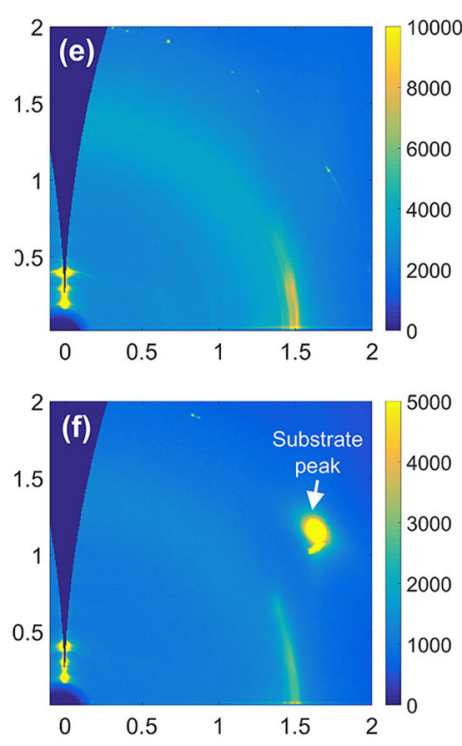
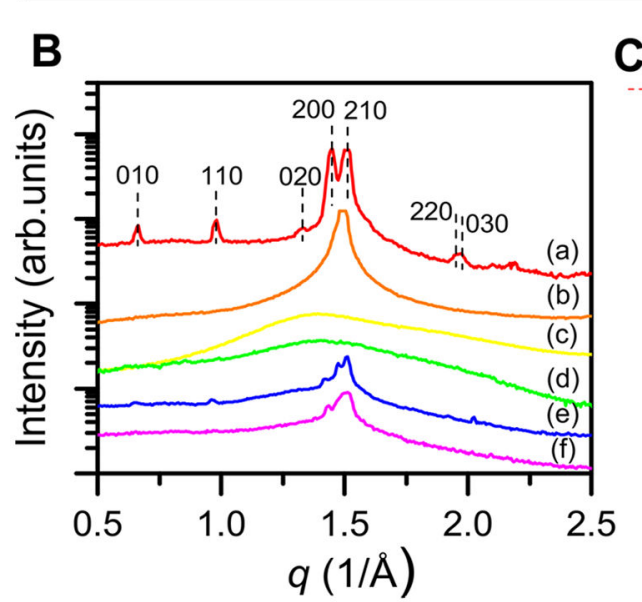

C --- When all lipids form a gel phase

- Calculated from experimental data
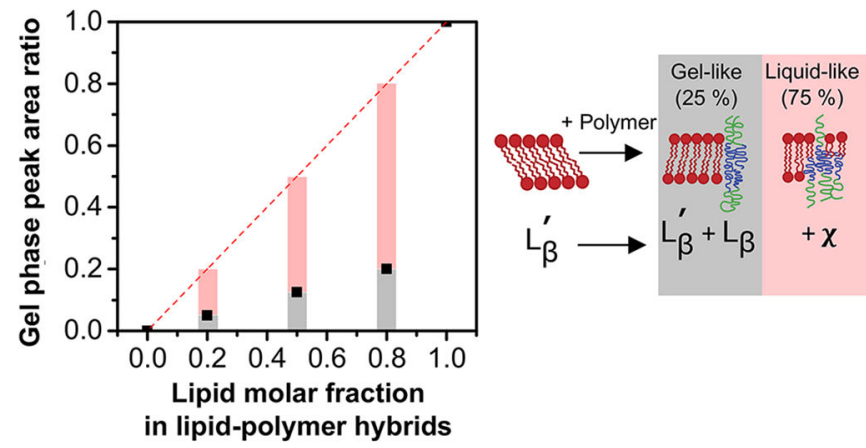

Figure 4.

(A) GIWAXS two-dimensional reciprocal space images of the films: (a) DPPC, (b) DPPC +5 mol \% PTX, (c) PBD- $b$-PEO, (d) PBD-b-PEO + 5 mol \% PTX, (e) 4:1 molar ratio of DPPC to PBD- $b$-PEO hybrid, (f) 4:1 molar ratio of DPPC to PBD-b-PEO hybrid +5 mol \% PTX. Note the difference between $(a, e)$ and others; $(a, e)$ show several rod-shaped in-plane diffraction signals, while (b, f) display a single in-plane signal and (c, d) show no in-plane diffraction. (B) GIWAXS one-dimensional I $(q)$ profiles of (a-f) films to compare the Bragg reflections along the in-plane direction $\left(q_{x y}\right)$. In (a), Bragg peaks assigned to a lipid headgroup unit cell are indicated by vertical dashed lines and their associated Miller indices. (C) Left: from the GIWAXS data sets, the integrated intensity of the gel phase peak was calculated and the relative peak area ratios of hybrid films to pure DPPC films were calculated (denoted by black square points). If DPPC lipids maintain the gel phase not affected by the presence of PBDPEO, the area ratio should be proportional to the DPPC to PBDPEO molar ratio (denoted by the red dashed line), which was not the case of our 
systems. Right: an illustration summarizing lipid in-plane phase transitions upon polymer incorporation. When polymer is present, only $\sim 25 \%$ of the lipids remain as gel-like phase $\left(\mathrm{L}_{\beta}^{\prime}\right.$ and $\left.\mathrm{L}_{\beta}\right)$, while the other $\sim 75 \%$ turn into more disordered, liquid-like phase $(\chi)$. 

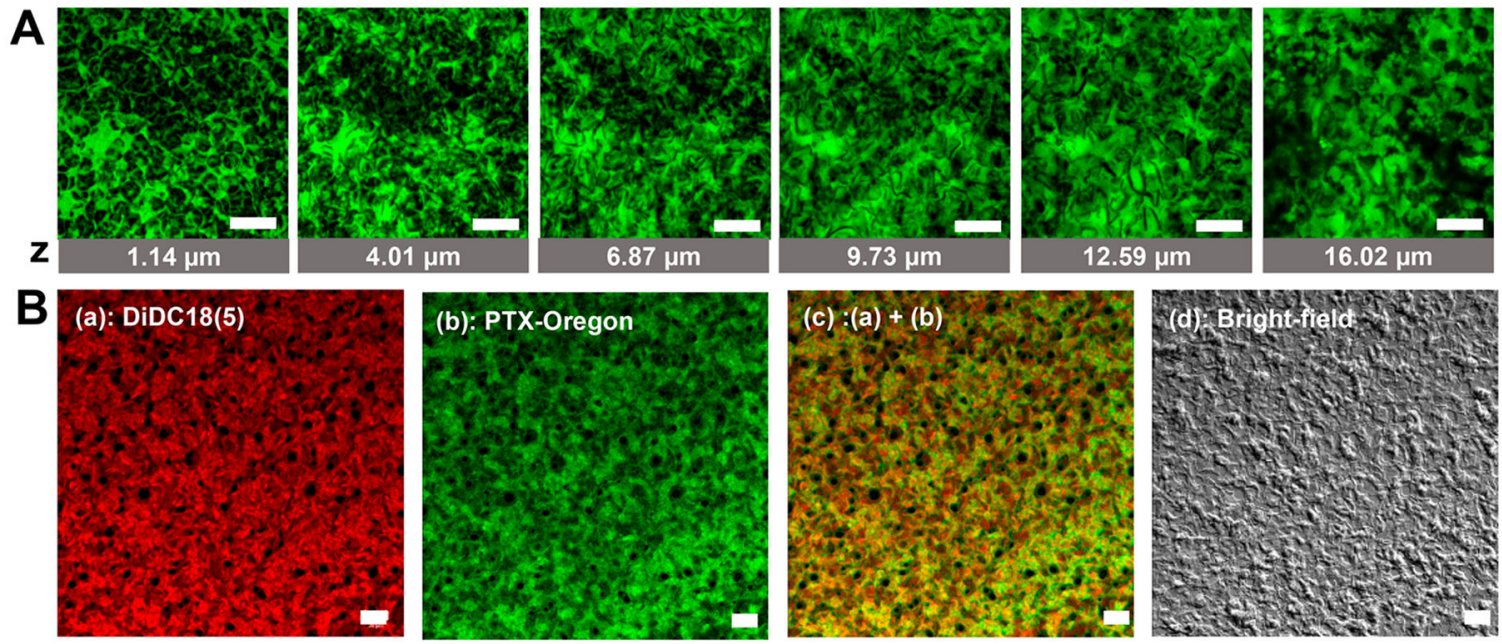

Figure 5.

CLSM images of hybrid films (1:1 molar ratio of DPPC to PBD- $b$-PEO +5 mol $\%$ PTX) in water, doped with PTX-Oregon $488(0.2 \mathrm{~mol} \%)(\mathrm{A})$ at selected depths and (B) at different channels. DiDC18(5) was added at $0.1 \mathrm{~mol} \%$. The distribution of PTX drugs in the films was heterogeneous both in-plane and out-of-plane. Scale bars $=20 \mu \mathrm{m}$. 

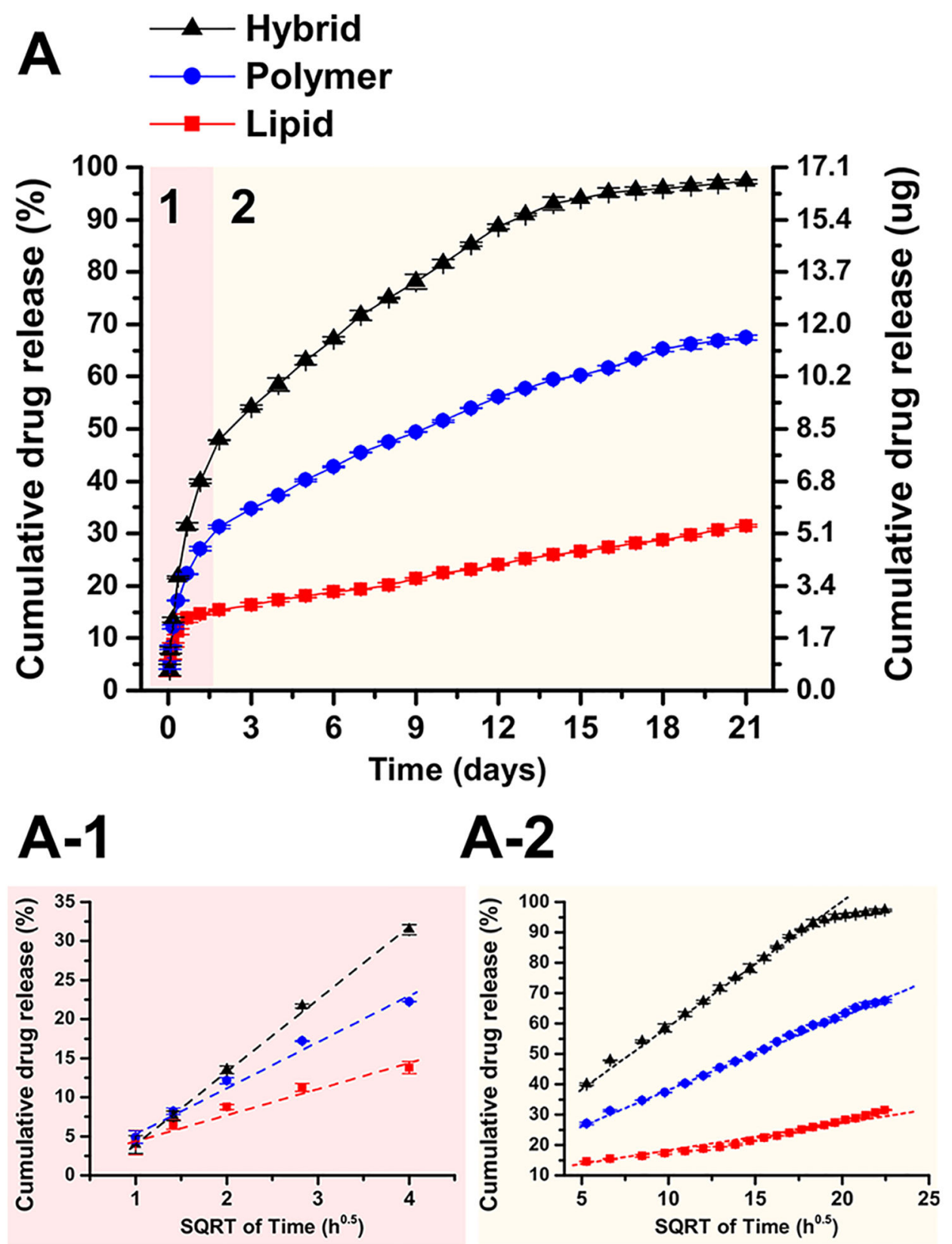

Figure 6.

(A) Cumulative release profiles of PTX from 1:1 molar ratio of DPPC to PBD- $b$-PEO hybrid (black triangles), PBD- $b$-PEO (blue circles), and DPPC (red squares) films with $2 \mathrm{~mol} \%$ PTX incorporated. The hybrid films showed significantly higher and faster PTX release compared to single-component control films. The PTX release profiles showed two characteristic release stages: initial fast release and slower release afterward. (A-1, A-2) Each stage of PTX release profile was plotted against the square root of time to show that PTX release follows diffusion-controlled kinetics (Higuchi model ${ }^{97}$ ). The dashed lines indicate the linear relationship between the square root of time versus cumulative drug release. All values are the arithmetic mean $\pm \mathrm{SD}, n=3$. 
2 theta $\left({ }^{\circ}\right)$

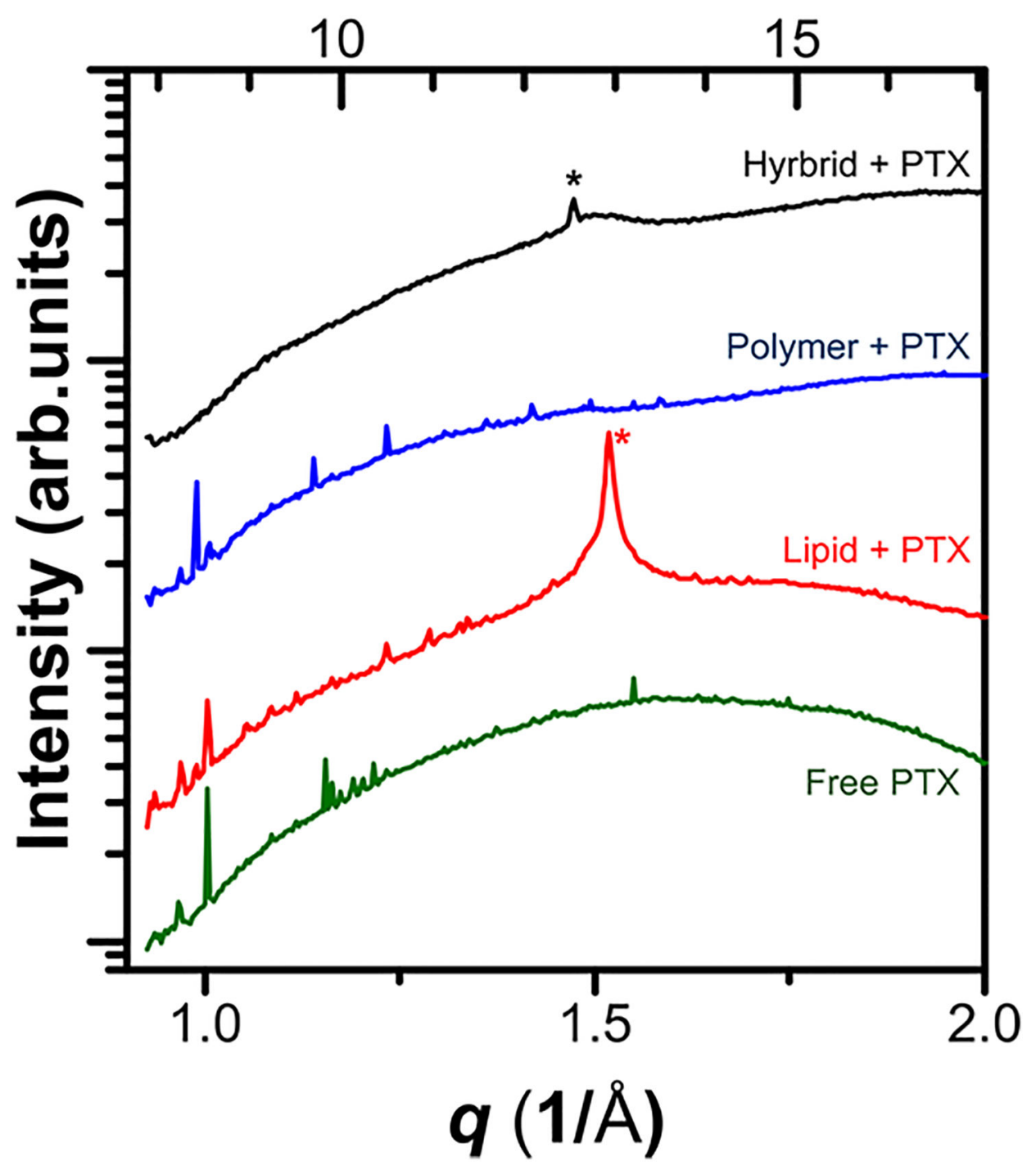

Figure 7.

Transmission WAXS one-dimensional I ( $q$ or 2 theta) profiles of hybrid vesicles (1:1 lipid to polymer molar ratio), liposomes, polymersomes with $5 \mathrm{~mol} \%$ PTX incorporated, and free PTX in excess water. Lipid and polymer samples showed sharp diffraction peaks associated with PTX crystals, indicating that single-component systems cannot efficiently entrap all the PTX molecules, resulting in undesirable PTX crystallization. Hybrid samples displayed no PTX crystal peaks. $(*)$ Peaks are from the lipid tail ordering. 
Table 1.

Results of Higuchi Models Applied to PTX Release Profiles of Lipid, Polymer, and Hybrid Films ${ }^{a}$

\begin{tabular}{lcccc}
\hline & \multicolumn{2}{c}{ stage 1 } & \multicolumn{2}{c}{ stage 2 } \\
\hline sample & $\mathbf{K}_{\mathbf{H}}\left(\mathbf{h}^{\mathbf{- 1 / 2}}\right)$ & $\boldsymbol{R}^{\mathbf{2}}$ & $\mathbf{K}_{\mathbf{H}}\left(\mathbf{h}^{\mathbf{- 1 / 2}}\right)$ & $\boldsymbol{R}^{\mathbf{2}}$ \\
lipid & 3.112 & 0.9706 & 1.011 & 0.9734 \\
polymer & 5.764 & 0.9866 & 2.424 & 0.9979 \\
hybrid & 9.264 & 0.9986 & 3.984 & 0.9978 \\
\hline
\end{tabular}

${ }^{a} R^{2}$ is correlation coefficient and $K_{\mathrm{H}}$ is the Higuchi release rate constant calculated from the slopes of the respective plots. 